\title{
Mindfulness Promotes Control of Brain Network Dynamics for Self-Regulation and Discontinues the Past from the Present
}

Dale Zhou ${ }^{1}$, Yoona Kang ${ }^{2}$, Danielle Cosme ${ }^{2}$, Mia Jovanova ${ }^{2}$, Xiaosong $\mathrm{He}^{3,4}$, Arun

Mahadevan $^{3}$, Ovidia Stanoi ${ }^{5}$, Julia K. Brynildsen ${ }^{3}$, Nicole Cooper ${ }^{2}$, Eli J. Cornblath ${ }^{3}$, Linden Parkes ${ }^{3}$, Peter J. Mucha ${ }^{6}$, Kevin N. Ochsner ${ }^{5}$, David M. Lydon-Staley ${ }^{2,3,7}$, Emily B. Falk $^{2,8,9}$, and Dani S. Bassett ${ }^{3,10-15}$

${ }^{1}$ Neuroscience Graduate Group, Perelman School of Medicine, University of Pennsylvania, Philadelphia, PA 19104, USA

${ }^{2}$ Annenberg School for Communication, University of Pennsylvania, Philadelphia, PA 19104, USA

${ }^{3}$ Department of Bioengineering, School of Engineering and Applied Sciences, University of Pennsylvania

${ }^{4}$ Department of Psychology, School of Humanities and Social Sciences, University of Science and Technology of China, Hefei, P.R. China

${ }^{5}$ Department of Psychology, Columbia University, New York City, NY, USA ${ }^{6}$ Department of Mathematics, Dartmouth College, Hanover, NH, USA

${ }^{7}$ Leonard Davis Institute of Health Economics, University of Pennsylvania, Philadelphia, PA, USA

${ }^{8}$ Department of Psychology, University of Pennsylvania, Philadelphia, PA, USA

${ }^{9}$ Marketing Department, Wharton School, University of Pennsylvania, PA, USA

${ }^{10}$ Department of Physics \& Astronomy, College of Arts and Sciences, University of Pennsylvania

${ }^{11}$ Department of Neurology, Perelman School of Medicine, University of Pennsylvania ${ }^{12}$ Department of Electrical \& Systems Engineering, School of Engineering and Applied Sciences, University of Pennsylvania

${ }^{13}$ Department of Psychiatry, Perelman School of Medicine, University of Pennsylvania, Philadelphia, PA, USA

${ }^{14}$ Santa Fe Institute, 1399 Hyde Park Rd, Santa Fe, NM 87501 USA

${ }^{15}$ To whom correspondence should be addressed: dsb@seas.upenn.edu

December 3, 2021 


\begin{abstract}
Mindfulness is characterized by attentiveness to the present experience with nonjudgmental awareness and acceptance. Practicing mindfulness alters brain function to support the executive regulation of thoughts, feelings, and behavior. While early stages of practice are thought to require greater "neural effort" for later efficiency, current evidence relies on circular definitions of effort based on functional activity magnitude. Here we used network control theory as a model of how external control inputs, which operationalize effort, can distribute changes in neural activity across the macro-scale structural brain network. Further, we inferred the intrinsic timescale of activity to operationalize present-centered activity as shorter momentary timescales that discontinue the past and update the present. To explain effects of mindful regulation on alcohol consumption, we applied these methods to a randomized controlled intervention study with resting-state and task fMRI data. The task primed participants to either mindfully respond or naturally react to alcohol cues. Mobile text interventions and measurements of alcohol consumption were administered using ecological momentary assessments during the subsequent 4 weeks. We hypothesized that neural states of mindfulness require greater effort to enact and sustain. This effort may support deautomatized habitual natural reactions, discontinued processing, and updated present-centered neural dynamics. We found that mindful regulation of alcohol cues, compared to the natural reactions of the benchmark group, involved more effortful control of neural dynamics across cognitive control and attention networks. This effort persisted in the natural reactions of the mindful group compared to the benchmark group. Using resting-state fMRI, we found that more effortful neural states tended to occur over shorter timescales than less effortful states. Our findings provide an explanation for how neural dynamics with altered effort and stability, such as mindful states, tend to center the present experience.
\end{abstract}


In many situations of daily life, humans may desire to engage in some form of self-regulation. Key to selfregulation is the idea of marshalling resources to achieve a goal 1, 2, 3, Mindfulness is an increasingly taught regulatory strategy to support people's ability to achieve educational, work, and health goals [4, 5, 6, 7, 8, 9, Although different definitions and strategies for engaging mindfulness likely involve different cognitive processes, the state of mindfulness is commonly characterized by attentiveness to the present experience with nonjudgmental awareness and acceptance [10, 11, 12, 13, 14]. Understanding the neural bases of mindfulness is important because identifying neural processes that are characteristic of mindfulness can help determine who is likelier to benefit from practice, inform clinical applications, and guide intervention programs [15, 16]. This understanding of neural processes is particularly essential if those neural processes rely on brain regions that are not yet developmentally mature or are disrupted in mental illness [17, 18, 19. However, past research has been limited in its ability to explain the neural processes relating mindfulness and self-regulation 14.

Key challenges in the field derive from the fact that mindfulness requires practice, and as one practices, one transitions from exerting effort to more efficient performance [20, 14. In comparing novices and experts, evidence indicates that early stages of mindfulness practice involve neural effort characterized by increased neural activity in regions associated with cognitive control, prospecting about the future, and thinking about the self; advanced stages involve decreased neural activity in those regions, suggesting an emerging neural efficiency [20, 14]. This observation raises three issues that limit our ability to explain the neural processes relating mindfulness and self-regulation. First, it is challenging to disentangle the functional significance of efficiency and that of effort because a key goal of mindfulness practice is to more efficiently engender less automatic reactions to one's experiences [21, 22, 23, 19, 24. Second, current theoretical definitions of neural effort and efficiency are circular because they are based on functional activity magnitude. Effort and efficiency are imprecisely defined for specific behaviors and contexts, resulting in an underconstrained interpretation of activity magnitude [17, 25]. With both increased and decreased activity within brain regions associated with different categories of meditation [26], high activity levels need not correspond to effort and low levels need not correspond to efficiency because decreasing activity can require the exertion of neural effort for inhibition [27, 28. Third, a core element of the definition of mindfulness relates to momentary timescales - present-centered awareness - but current analyses of neural activity focus on average statistical associations that obscure time [11, 14]. Building upon the progress of region-of-interest studies [14, we can address these issues by modeling the time-dependent control of dynamics on brain networks [29, 30] to better 
understand the neural processes of mindfulness and self-regulation.

Here, we operationalized the effort, efficiency, stability, and present-centeredness of neural dynamics during mindful self-regulation. Our operationalizations leveraged the framework of network control [31, 32, 33, wherein nodes represent brain regions with a given level of activity and edges represent structural connections along which activity flows. As in prior work, we used network control theory to determine how underlying white matter architecture constrains transitions between different neural states [34, 35, 36, 37]. A neural state is a single instance of activity across all brain regions; a neural transition is the change from an initial neural state to a target neural state; and a neural trajectory is a series of transitions between neural states over time. The controllability of a dynamical system describes the possibility of driving neural trajectories with external and time-dependent control input to all or a subset of brain regions [38. With this conceptual architecture in hand, we can posit physical and mathematical operationalizations of effort, efficiency, stability, and present-centeredness.

First, we operationalize effort and efficiency. When greater control input is required to execute a neural transition, we say that the transition is more effortful [39, 36, 34, whereas when less control input is required, we say that the transition is more efficient. The amount of control input needed depends on the trajectory of endogenous, or natural, activity that propagates across the structural network; more control input is needed to steer endogenous trajectories that naturally stray from a target neural state than to steer those that tend toward the target state [38. Control inputs are positively correlated with working memory load and are modulated by dopaminergic signaling [34, a neurotransmitter that encodes information about a task's cognitive demand, effort, and expected benefit [40, 41. Since the diversity of neural transitions depend on structural connectivity [42, here we also consider the average controllability as an operationalization of the efficiency of all possible neural trajectories solely from network structure rather than only considering single empirically observed trajectories. Average controllability quantifies the capacity of the control inputs into each brain region to distribute activity across the structural network and guide changes in activity to any new hypothetical states [39. Regions with high average controllability are more influential in efficiently controlling network dynamics across multiple cognitive domains [38], are heritable [43], and are over-represented in the default mode network [39], which has been hypothesized to garner efficiency gains from expertise in mindfulness [20, 14. The average controllability across regions is positively correlated with activity during tasks that probe executive function [44. Executive function is defined as the capacity to 
coordinate resources of cognitive control, attention, memory, and inhibition towards achieving goals. As a regulatory process that supports achieving goals, mindfulness may share some relation with executive function [45, 5, 46, 14, 44, 47]. Hence, we use network control theory to understand the relationships between executive function, mindfulness, and self-regulation.

With the metric of control inputs, we now have a theoretical framework to operationalize the stability of neural dynamics. This operationalization of stability will in turn allow us to build intuitions of how the metrics of effort and efficiency — and relationships with executive function, mindfulness, and self-regulation-all pertain to our operationalization of the present-centeredness of neural dynamics. The stability of neural dynamics is operationalized as inversely proportional to the control input required to transition from a given neural state to itself [38]. This definition assumes that more "energetic" or costly states are more likely to change to different states [34, consistent with constraints on the expenditure of limited resources [48, 49]. In light of this constraint, it is preferable for the brain network to discontinue rather than persist in costly neural states. Discontinuing a state is updating it, an intuition that characterizes present-centeredness. Accordingly, we operationalized the present-centeredness of neural dynamics as the duration for an initial neural state to update to a different (less correlated) neural state, a metric called the intrinsic timescale [50, 51. If past neural states more quickly subside, then the present neural state may be more distinct from past states. The more quickly states update to new states, the more present the dynamics. Modeling and analyzing dynamics across time allows us to provide a physical and mathematical operationalization for the subjective phenomenological concept of staying in present [11, 19, 14].

Using these measures of effort, efficiency, stability, and present-centeredness, we investigated the neural dynamics of mindfulness in a dataset of 76 college students randomized into either a mindfulness or benchmark group assigned to react naturally. College students consume more alcohol than peers who do not attend college, leading to negative academic, social, and legal outcomes [52. General self-regulation ability, without specific goals, is linked with behavior change in alcohol consumption and attenuates the consequences of that consumption [53, 2]. Hence, we measured changes in alcohol consumption as a real-world outcome of novices to the practice of mindful self-regulation. In the fMRI scanner, the mindfulness group was instructed to react to images of alcohol either mindfully or naturally, whereas the benchmark group was only instructed to react naturally. During mindful trials, participants were instructed to mentally distance themselves by observing the situation and their response to it with a more impartial, nonjudgmental, or curious mindset, 
and without getting caught up in the situation or response. During natural reaction trials, participants were instructed to simply look and respond according to initial gut reactions, without thinking of anything in particular. During the subsequent 4 weeks, we used ecological momentary assessments to expose participants to text interventions reminding them either to react mindfully or naturally and without explicit instruction to moderate alcohol consumption. These assessments also served to measure self-regulated drinking behavior. To investigate the differences between neural trajectories of the mindfulness and benchmark groups, we calculated the control inputs that the frontoparietal control and dorsal/ventral attention subnetworks should exert to drive brain function (1) from the baseline state to the task state, and (2) from the task state to itself. We focused on the regions in these subnetworks because they have been associated with cognitive control, regulatory processing, and constraints on mindwandering [14, 26, 54]. To investigate the intrinsic timescale of neural dynamics, we analyzed resting-state fMRI scans that were acquired prior to the exposure to mindfulness. We were interested in the resting-state for two reasons. First, activity at "rest" is relevant for self-regulation due to fewer constraints imposed by external stimuli and incentives [55, 18]. Rather the resting-state activity is more related to prospecting about the past or future, mindwandering, and thinking self-referentially [21]. Second, the effects of meditative practice are thought to persist by transforming the resting-state experience to be more present-centered [20. In the context of our experiment, the effect of mindful practice on effort and stability may persist to impact how individuals naturally react. Therefore, the activity during the resting-state and natural reactions, measured without any explicit task or thinking of anything in particular [56], provide an opportunity for a test of the generalizability of changes in effort, efficiency, and stability elicited by mindfulness training to impact natural reactions.

Here, using these methods and data, we test four hypotheses that posit relationships between executive function, mindfulness, and self-regulation, linking them to the concept of present-centeredness. Our first hypothesis stems from recent evidence that mindful regulation predicts later moderation of alcohol consumption despite no specific instruction to do so (manuscript in preparation). First, given the similarly supportive role of executive function and mindfulness on self-regulation [11, 24, 14, we hypothesized that the average controllability of the whole structural network - an operationalization of executive function - would predict later moderation of alcohol consumption across all individuals. Our second hypothesis draws upon a model of mindfulness wherein deautomatization is a mechanism by which mindfulness promotes self-regulation [24]. Deautomatization is the discontinuation of emotional and cognitive reactions that have become habitual and spontaneous. In light of this potential deautomatizing function of mindfulness, our second hypothesis was 
that - compared to the benchmark condition - participants in the mindful condition performing mindful regulation would exert more effort that would also persist to impact their natural reactions, suggesting effort to deautomatize habitual reactions to alcohol [57, 24]. Our third hypothesis was motivated by the concept of non-elaborative processing, which is a major component of deautomatization [24]. Non-elaboration is defined as the cessation of one's conceptual analysis of expectations and experiences, such as the ruminative cycles of negative and self-focused thoughts which can compound physical or psychological pain [58. Non-elaboration can help to create distance from mental events and emphasize their transience, separating one's perception from one's response or evaluation [59, 60]. Our third hypothesis was that if mindfulness practice involved the cessation of neural states associated with alcohol cue processing towards new neural states, then the neural states of the mindful group would be more unstable than those of the benchmark group. With our fourth and final hypothesis, we aimed to investigate how executive efficiency, effort, and stability support the goal of mindfulness to center the present [11, 24]. Transitioning to different states, in general, with efficiency, effort, or instability may be related to discontinuing past states and updating the present state. Hence, our fourth hypothesis was that brain regions with greater average controllability and resting-state trajectories with greater effort and instability would have quicker intrinsic timescales reflecting present-centered activity 61, 14. In testing these four hypotheses, our study elucidates how the control of brain network dynamics permitted by structural network controllability or elicited during mindful regulation support self-regulation and center the present.

\section{Results}

\section{Average controllability predicts later behavior change in alcohol consumption}

We sought to apply network control theory to investigate how mindfulness influences brain dynamics to support self-regulation (Figure 1). Prior findings in this dataset indicate that individuals randomly assigned to the mindfulness or benchmark conditions differed in their self-regulation of alcohol consumption. Specifically, mindfulness promoted moderation of alcohol consumption (manuscript in preparation), consistent with other reports of mindfulness improving executive control and self-regulation 63. In addition to this finding of differences between groups, we first hypothesized that individuals across groups would moderate alcohol consumption by reducing drinking probability if their structural networks afforded greater average controllability. Average controllability is a measure that characterizes the capacity of brain regions to efficiently drive activity across structural connections to any hypothetically reachable state (Figure 2A). To model 
A

\section{Self-regulatory process}

Mindfulness

$(n=37$; benchmark group $n=39$ )

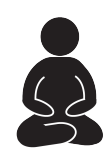

B
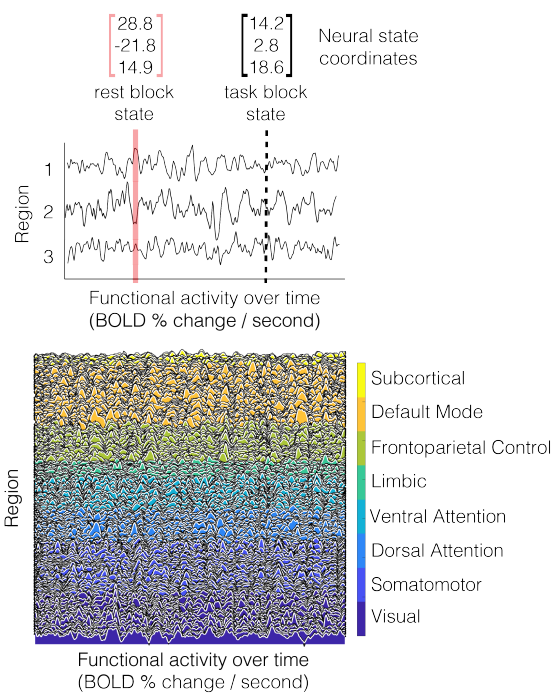

Executive function

Network control of structurally constrained neural dynamics

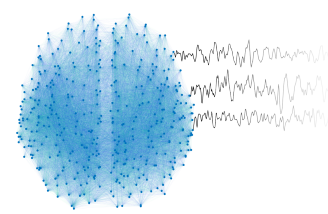

Behavior change

Alcohol moderation

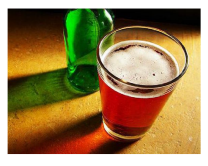

C
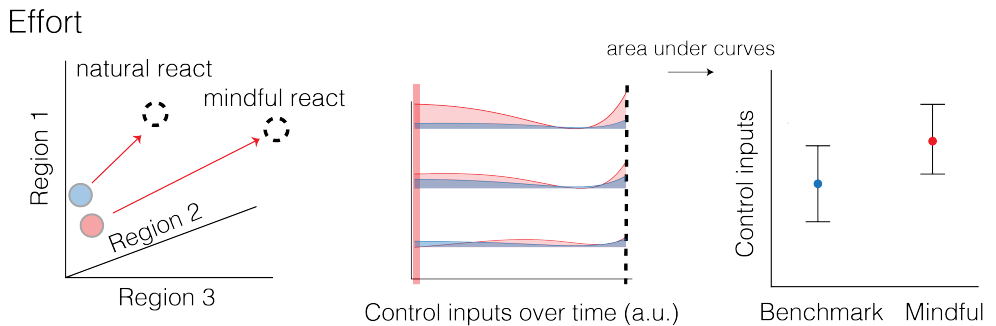

Stability
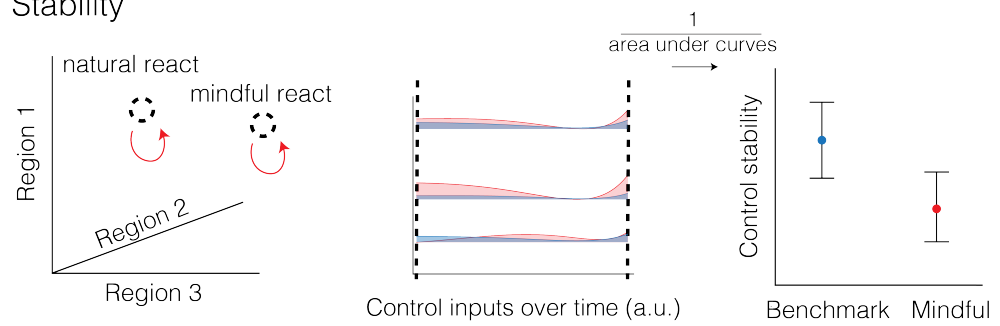

Figure 1: Control of brain network dynamics for regulatory strategies and behavior. (A) The current study aims to understand the effort, efficiency, and stability of neural dynamics during mindful states. Mindful regulation of alcohol cues may support self-regulation of alcohol consumption. Images were used from the Galician Beverage Picture Set [62]. (B) The conceptual schematic depicts a simplified brain with only 3 regions to explain the intuition underpinning the control metrics of effort and stability. The measured fMRI activity time course of these 3 regions defines only one of many possible activity trajectories that those regions could have produced. We use network control theory to calculate the control inputs required to steer functional states from an initial state (pink line) propagating according to simulated linear dynamics atop the structural connectome towards a target state (dashed black line). We apply the same framework to analyze the full time course of 400 cortical and 14 subcortical regions. A neural state is defined as a single instance of activity across all 414 brain regions, measured by repeated scan acquisitions. All neural states measured across time compose the trajectory of the neural dynamics. (C) Here we depict two of the main hypotheses regarding the effort and stability of mindful states. In keeping with the simplified 3-region system, the system's state can be visualized as a coordinate in 3-dimensional space. To determine the effort of neural dynamics, we calculated the optimal control inputs required to transition (1) from the baseline to the natural reaction state, and (2) from the baseline to the mindful reaction state. To determine the stability of neural dynamics, we calculated the optimal control inputs required to sustain the natural reaction state and sustain the mindful reaction state. We then compared the control input and stability between the benchmark and mindful conditions. 
A

\section{Average Controllability} all theoretical state transitions possible from 1 unit of input

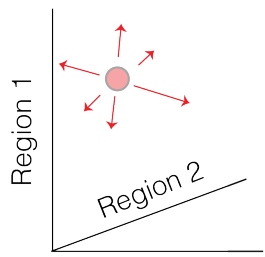

Region 3
B

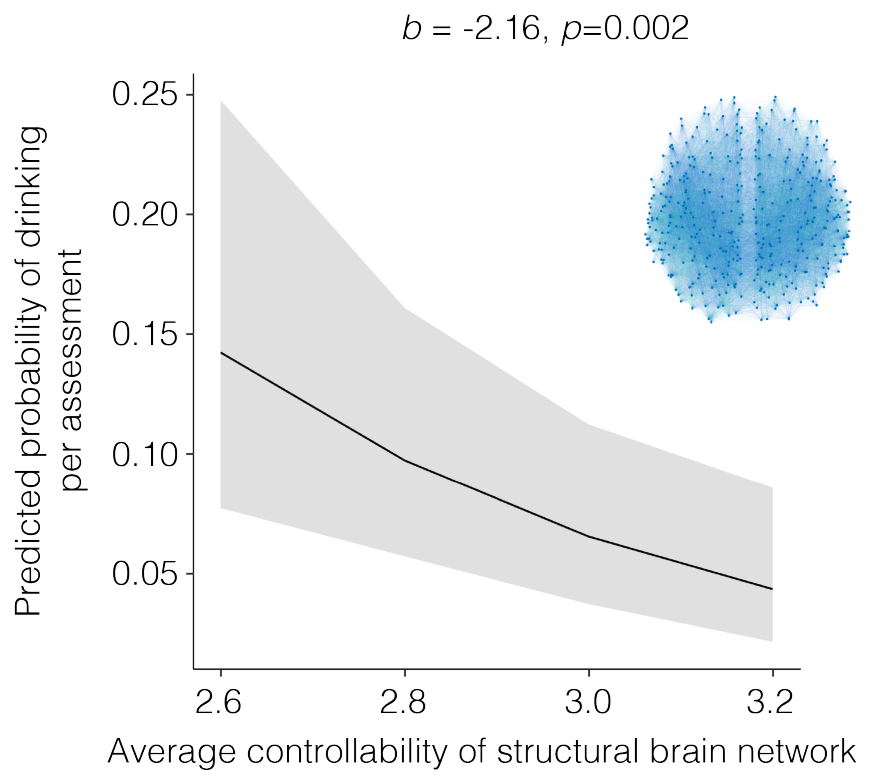

Figure 2: Average controllability predicts later behavior change in the moderation of alcohol consumption. (A) Here we visualize 6 possible changes in neural activity (red arrows depict change to a target state) for three brain regions. Average controllability is a measure of the capacity of the structural connectome to drive all new brain states (all target states on the surface of the ellipsoid). (B) For each individual structural brain network, we obtained an individual measure of average controllability as the mean average controllability across all brain regions (inset). Among all individuals, structural connectivity that supported more distant hypothetical neural trajectories predicted later reductions in the observed probability of consuming alcohol on a given occasion. The plot displays predicted values of the marginal effects of average controllability using a multi-level regression model. 
the logistic drinking probability of each individual at a given occasion, we built upon a recent report that used a multilevel model with zero-inflation to account for repeated measures, individuals nested within social groups, and the non-normally distributed drinking data with the predominance of assessments that reported zero drinking [64] (manuscript in preparation). In this recent work, covariates were included in the model to control for the number of assessments, the number of responses to alcohol surveys, whether the day of assessment was a social week day [65, and whether individuals were exposed to mindfulness prompts that week. In testing for the effects of average controllability of the structural brain network across all participants, we additionally controlled for the baseline drinking amount or frequency in the past 6 months, demographic variables, and the personality trait of attentional impulsivity (see Methods section 'Statistical modeling' for details). Across both the mindful and benchmark conditions, we found-with the zero-inflated model of drinking probability - that individuals with greater average controllability across all regions tended to have a lower probability of drinking per assessment beyond pre-existing differences in their baseline drinking frequency (Figure 2B; $b=-2.16, p=0.002$ ). This observation is consistent with our hypothesis and with previously reported associations between average controllability and executive function [44. In sum, mindfulness interventions and baseline average controllability each support later behavior change in the moderation of alcohol consumption.

\section{Mindfulness deautomatizes and discontinues neural states of alcohol cue reaction}

The practice of mindfulness is typically thought to be effortful for novices [14. Here, in addition to considering all possible hypothetical trajectories by calculating the average controllability, we also sought to understand how mindfulness supports self-regulation by comparing the specific empirical neural trajectories of the mindfulness group to the neural trajectories of the benchmark group during the fMRI task. To investigate these neural trajectories, we calculated the control inputs that the frontoparietal control and dorsal/ventral attention subnetworks should exert to drive brain function from baseline states to task states. Baseline states were defined as a neural state with zero task-related activity. Task states corresponded to the neural states of task-related activity across trials which instructed individuals in the mindful condition to react to alcohol cues mindfully, or trials which instructed them to react naturally (Figure 3A). Task states for individuals in the benchmark condition corresponded only to trials instructing them to react naturally to alcohol cues.

Our second hypothesis was that the deautomatizing function of mindful practice would evoke neural states 

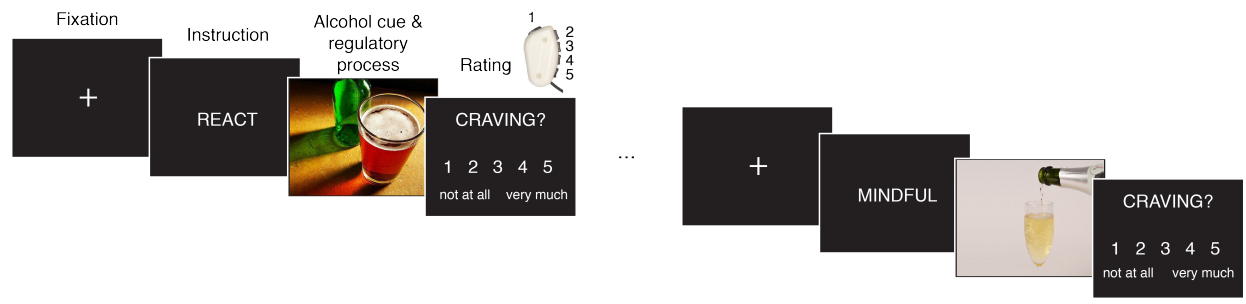

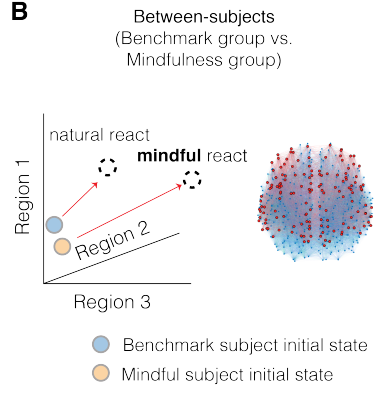

D

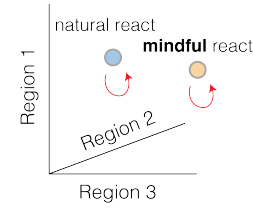

Benchmark subject initial state Mindful subject initial state

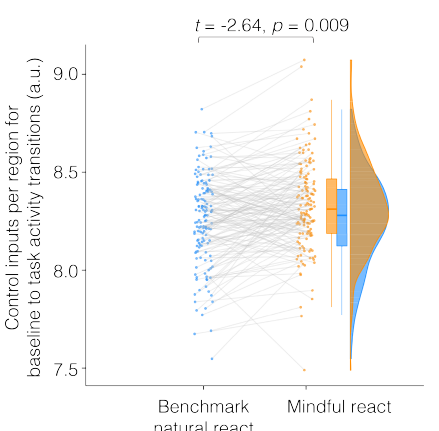

C

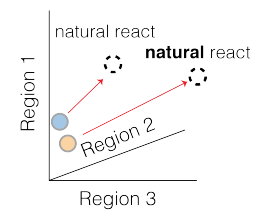

Benchmark subject initial state

Mindful subject initial state

E

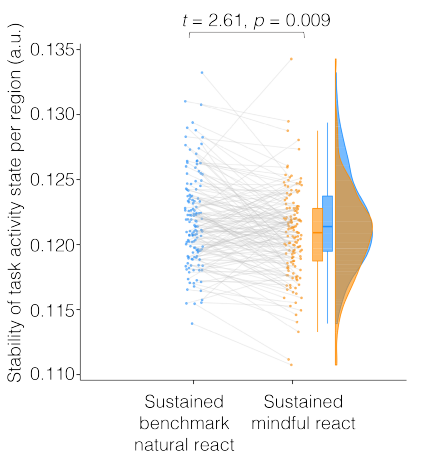

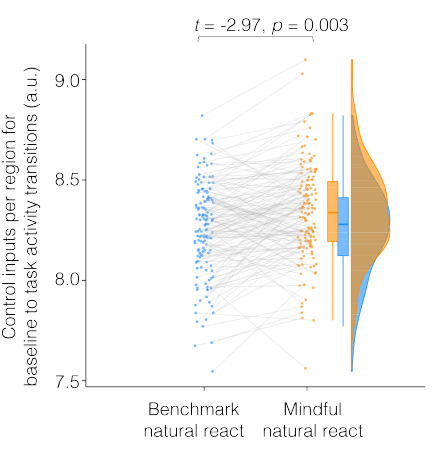

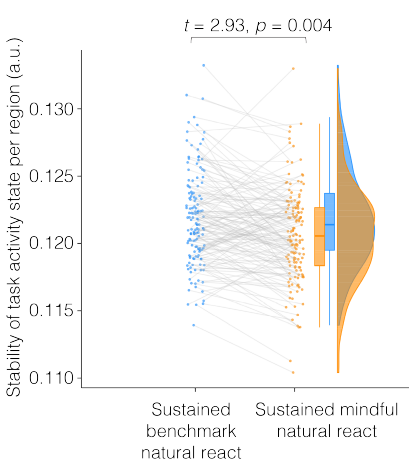

Figure 3: Mindfulness deautomatizes and discontinues neural states of reacting to alcohol cues. (A) A trial instructing natural reactions and a trial instructing mindful regulation. The task included 96 total trials arranged in blocks of 4 with the same regulatory strategy. We were interested in the effort and stability of neural states during the mindful regulatory process compared to the natural reaction. (B) Executive control brain circuitry is thought to comprise the frontoparietal control, dorsal attention, and ventral attention subnetworks with 145 regions (colored red). We compared mindful reactions to natural reactions of the benchmark group when only allowing control inputs to regions of the brain associated with cognitive control. We found that individuals who were randomly assigned to the mindfulness condition exerted more control for brain function when mindfully reacting to alcohol cues than controls did when naturally reacting. Grey lines connect the same brain region between the benchmark and mindful groups. (C) We next calculated the control inputs required to persist in the neural state of mindful regulation or natural reaction. The neural states corresponding to mindful reactions in the mindful condition were less stable than the neural states corresponding to natural reactions in the benchmark condition. (D) Here, we compared natural reactions of the mindful group to natural reactions of the benchmark group when only allowing control inputs to regions of the brain associated with cognitive control. We found that the individuals in the mindfulness condition still exerted more control for brain function when naturally reacting to alcohol cues than controls did when naturally reacting. (E) The neural states corresponding to natural reactions in the mindful condition were less stable than the neural states corresponding to the natural reactions in the benchmark condition. Together, greater control inputs suggest greater effort, consistent with the hypothesis that mindfulness effectively supports self-regulation by deautomatizing reactions. Reduced stability of the neural states of mindful and natural reactions in the mindful condition suggest brain function that discontinues and dwells less on states of reaction. 
that require more effort and this effort would also persist into conditions requiring the individual to react naturally, suggesting effort related to the deautomatization of habitual reactions to alcohol. Consistent with our hypothesis, we found that the control inputs to these regions for individuals who reacted mindfully in the mindful condition were greater than inputs required to react naturally for individuals in the benchmark condition (Figure 3B; $t(288)=-2.64, p=0.009)$. This pattern of findings suggests that natural dynamics are deautomatized using control inputs during mindful regulation. Individuals who mindfully regulated required greater control input to the frontoparietal control and dorsal/ventral attention subnetworks on trials instructing them to react naturally than individuals in the benchmark condition who were similarly instructed (Figure 3C; $t(287.6)=-2.97, p=0.003)$. The neural states associated with mindful reactions to alcohol required greater control input than the natural reactions to alcohol of the benchmark condition. This effort persisted into conditions that required individuals to react naturally, suggesting a marked deautomaticity of neural dynamics.

Next, we tested the third hypothesis that the mindful states and the natural states of the mindful group would be more unstable than the natural reactions of the benchmark group. Evidence supporting this hypothesis would suggest that mindfulness practice elicits instability and the cessation of the current costly neural state towards new neural states. To test this hypothesis, we calculated the stability of the neural state as the reciprocal of the control input required to transition from the state to itself. Consistent with our hypothesis, we found that the neural states of regions in the frontoparietal control and dorsal/ventral attention subnetworks were less stable for mindful reactions than for natural reactions in the benchmark condition (Figure 3D; $t(287.82)=2.61, p=0.009)$. Reduced stability of the neural states of mindfulness suggest future discontinuation of the costly state of processing. If natural reactions of the mindful group were more unstable than natural reactions of the benchmark group, then the effort of mindful regulation that persisted to natural reactions may promote cessation of attending to the alcohol cues due to increased cost. We found that natural reactions of individuals who practiced mindful reactions were less stable than the natural reactions of individuals in the benchmark condition (Figure 3E; $t(287.99)=2.93, p=0.004$ ), suggesting greater cessation of the neural states associated with attending to the alcohol cues. Consistent with learned deautomaticity across both mindful and natural reactions, we did not find within-person differences in either effort or stability between the trials of mindful and natural reactions for the mindful group Supplementary Figure 1. Taken together, the greater effort and deautomaticity of reactions accompany more unstable neural states suggestive of discontinued attendance to alcohol cues. 


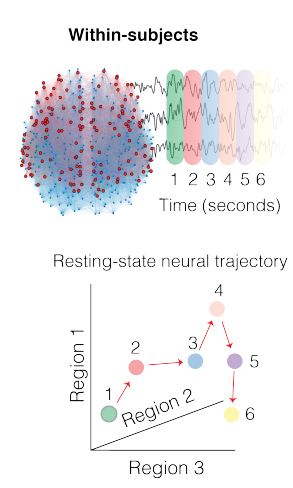

C

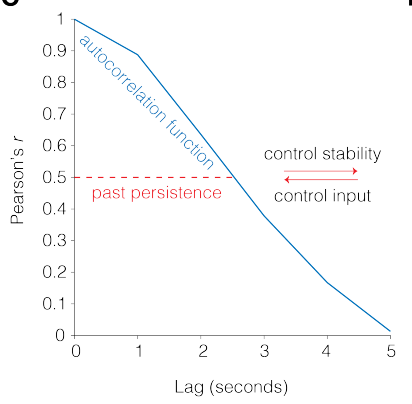

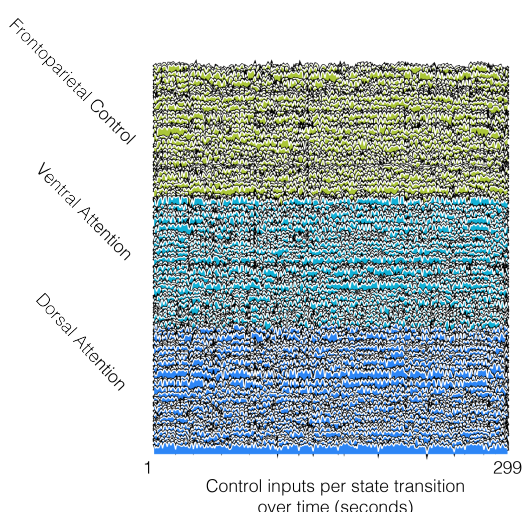

D

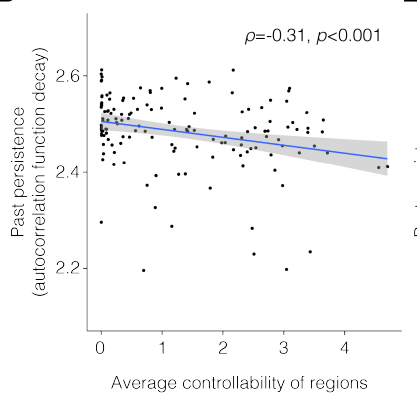

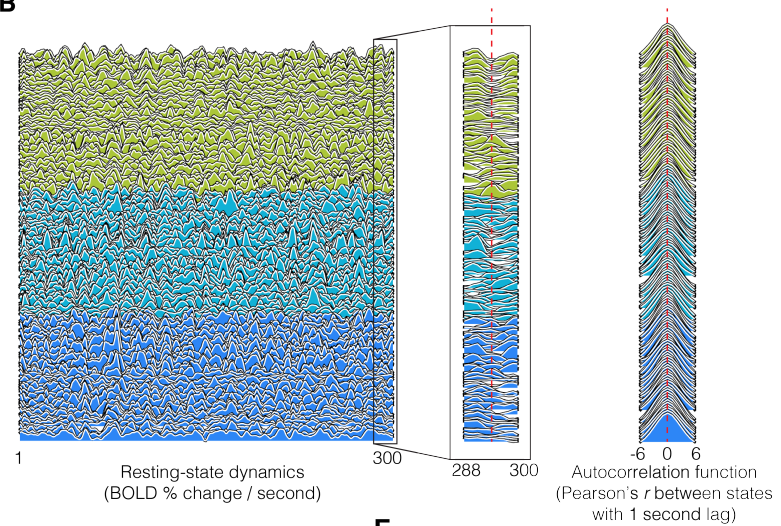

E

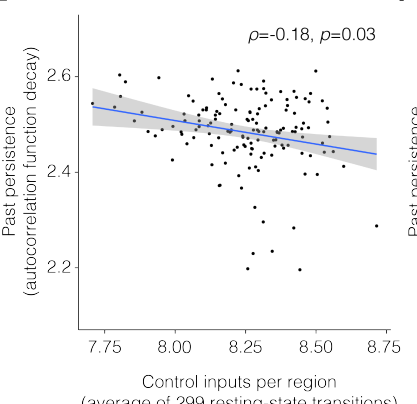

$\mathbf{F}$

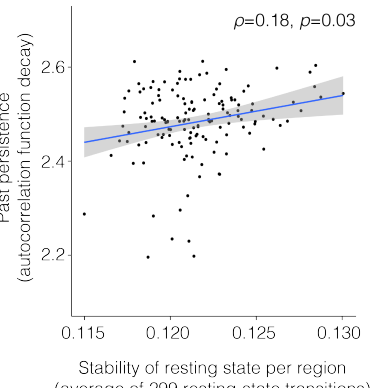

Stablity of resting state per region
(average of 299 resting-state transitions)

Figure 4: Deautomatization and discontinuation of resting-state trajectories are associated with more distinct past, present, and future states. (A) This schematic depicts the control inputs to cognitive control and attention regions that are required to transition from state to state (red arrows) as measured by the repetition time of each image in the resting-state time course. A state's activity across all regions was defined using the BOLD activity magnitude. We plot the control input values - to the executive systems per transition over time-that are theoretically required to steer all regions to a new state of activity. The resting-state is thought to characterize spontaneous brain dynamics of internally directed mind-wandering and past or future prospection. These spontaneous dynamics are less constrained by external stimuli. (B-C) Though neural dynamics display substantial variability, a brain region's neural states that occur in closer succession tend to be more similar. Conversely, states that occur farther apart tend to be more dissimilar. In addition to time between states influencing the (dis)similarity of neural states, control inputs could discontinue past states to update the present state. A primary aim of mindfulness is to cultivate a non-elaborative awareness of present experience and perceptual clarity of this awareness. We hypothesized that transitioning to different states, in general, may be related to discontinuing past states and updating the present state. More precisely, we hypothesized that brain regions with greater average controllability and resting-state trajectories with greater effort and instability would have quicker intrinsic timescales reflecting present-centered activity. The intrinsic timescale of a neural signal quantifies how long it takes for the signal at any given state to decay, regardless of particular content or mental object of the signal. The quicker a signal decays according to calculated temporal autocorrelation functions per region, the less similar one state is from the next and the less the past state persists into the present. (D) Regions with greater average controllability for more distant trajectories tended to exhibit more quickly decaying and dissimilar states. (E) Regions exerting greater control on brain function tended to have more quickly decaying and dissimilar dynamics. (F) Regions with less stable and more discontinuing states tended to exhibit more quickly decaying and dissimilar dynamics. Together, neural dynamics of cognitive control and attention networks that are characterized by deautomatization and discontinuation relate to neural trajectories with more distinct past, present, and future neural states. 


\section{Deautomatizing and discontinuing neural states promote the clarity of the past, present, and future during the resting-state}

In this final section, we sought to understand the functional relevance of neural dynamics that are characterized by effort and instability more broadly by investigating resting-state neural dynamics. A key goal of mindfulness is to stay in the present experience with non-elaborative awareness. Similar to prior work reporting changes in baseline functional connectivity in expert meditators [20, the consistency of effortful and unstable neural processes across both mindful and natural reactions in the mindful condition suggests that mindfulness alters brain function beyond the immediate practice. This persistence of the effects of mindfulness training has been proposed to transform the resting-state experience to one that is more present-centered [20]. Here we sought to understand if effortful and unstable neural states during resting-state fMRI across all participants were more present-centered. The resting-state scan was administered prior to exposure to the mindfulness task. Hence, the following analysis evaluates the broader claim that effortful and unstable neural states in general (mindfulness being one such state), tend to center the present.

We tested our fourth hypothesis that brain regions with greater average controllability and resting-state trajectories with greater effort and instability would have quicker intrinsic timescales consistent with presentcentered activity. For each individual, we calculated the regional average controllability to assess the ease of transitioning to any hypothetical new state, the optimal control inputs to assess the cost of the empirical resting-state trajectory (Figure $\mathbf{4 A}$ ), and the intrinsic timescale to assess the resting-state signal persistence over time (Figure 4B). The intrinsic timescale is the time that must elapse before the similarity between a current signal and past signal decays by half (Figure 4C). Differences in the intrinsic timescales across brain regions form a temporal hierarchy of neural processing whereby abstract information like goals can persist over longer durations than newly attended sensorimotor and contextual information [50, 51]. Here, we use the intrinsic timescale to measure the persistence of past state signals in order to operationalize "presentcentered activity." Shorter intrinsic timescales suggest present-centered activity that discontinues the past and updates the present [11, 5. As in the prior analyses, we focus on the regions of the frontoparietal, dorsal attention, and ventral attention subnetworks because prior work suggests that (i) executive resources direct task-unrelated thoughts towards personal goals, (ii) executive control processes coordinate the mindwandering state, and (iii) neural circuits of executive control and attention exert constraints on resting-state brain function [21]. 
Using these metrics, we first evaluated whether the ease of transitioning to any hypothetical state promotes neural dynamics that are more likely to evolve across states which are dissimilar. We found that regions of the frontoparietal control and dorsal/ventral attention subnetworks with higher average controllability tended to have dynamics with more dissimilar states and faster decay (Figure 4D; $\rho(142)=-0.31, p<0.001$ ). This observation suggests that average controllability and executive function might support regulatory processing through present-centered resting-state dynamics. Next, we assessed whether regions with resting-state trajectories that demand the structural network to exert greater control input per state transition will drive dynamics to more dissimilar and quickly decaying states. Consistent with this hypothesis, we found that regions which required greater control input to execute resting-state trajectories tended to have more dissimilar dynamics with quickly decaying states (Figure 4E; $\rho(142)=-0.18, p=0.03)$, suggesting greater control inputs tended to drive quicker transitions to more distant states. Lastly, we evaluated whether regions with greater control stability for sustained neural states would have slower decay and more similar dynamics than regions with lesser control stability. Consistent with this prediction, we found that regions with greater stability tended to have more similar dynamics with slower decaying states (Figure $\mathbf{4 F} ; \rho(142)=0.18, p=0.03$ ). This finding provides evidence that the reduced control inputs required to sustain the same state are related to the temporal stability of that state [66, 34]. These results suggest that present-centered activity is related to the structural controllability and functional control associated with executive function and mindful regulation.

\section{Discussion}

In summary, our findings suggest that mindfulness and executive control resources support the regulation of alcohol consumption by biasing the network control of neural dynamics that are more deautomatized, discontinuing, and present-centered. Average controllability predicted later moderation of alcohol consumption regardless of whether the participant was randomized into the mindful or benchmark condition, consistent with previous work reporting associations between average controllability and both executive function 44 and and emotion regulation [47. Compared to the benchmark participants who were naturally reacting, mindfulness tended to elicit greater control input expenditure into the cognitive control and attention networks in order to transition to the task-elicited neural state - across all brain regions - of mindfully regulating responses to alcohol cues. This increased expenditure occurred regardless of whether participants in the mindful group were instructed to react mindfully or naturally, suggesting that there exists a learned effort to deautomatize the habitual processing of natural reactions. We next sought to understand the func- 
tional relevance of these control metrics of efficiency or effort. Brain regions that could (greater average controllability) or did (greater control input) steer the neural trajectory to new and more distant states were associated with a resting-state experience that had shorter intrinsic timescales. This finding is consistent with present-centered neural dynamics that update and discontinue past states, regardless of the content or mental object of those states. This shared functional role of centering the present is consistent with previous evidence that awareness is central to mindfulness, unlike other forms of meditative practice where awareness could potentially hinder its efficacy [67, 5]. Prior work suggested that meditative practices alter the restingstate experience to be more present-centered [20]. Our results suggest that mindfulness may elicit neural states of non-elaborative attention to the present experience and cognitive distance from past reactions to external and internal stimuli by quickening the intrinsic timescale of neural dynamics to discontinue the past and update the present.

Our application of network control theory revealed neural processes distinctive of mindfulness that prime the expenditure of control resources by regions in the frontoparietal control and attention networks. Our results build upon progress made by region-of-interest studies which identified neural correlates of attention, cognitive control, and self-relevant future-oriented processing in support of personal goals [68, 61, 69]. The explanations offered by elucidating how pathways of activity flow are related to characteristics of neural processes align closely with the notion that reduced constraints by the brain networks associated with cognitive control and attention are likely to result in the absence of awareness of one's present mental state [21, 54, 70]. Our operationalizations of effort and stability also offer richer characterizations of mindfulness that accord to defining features including the non-elaborative awareness of the present [10]. The current findings add to prior literature that early stages of mindfulness meditation practice may improve the ability to orient attention by selecting specific information from multiple sensory stimuli and attending to conflicts between different regional activity [14]. In contrast, the inability to regulate attention is related to mindwandering [11]. Mindfulness is negatively correlated with mindwandering, consistent with the understanding of mindfulness as the awareness of the present experience and mindwandering as perceptual decoupling of attention and the external environment [71]. Both mindfulness and mindwandering appear useful in different contexts and recruit brain networks associated with executive and default mode function [11, 54]. Differing recruitment of brain regions according to context may generate constraints on automatic and deautomatized thoughts, feelings, and behaviors that vary in psychological components of intentionality, awareness, efficiency, and controllability [72. Our work further suggests a functional role of altered effort and stability 
during neural dynamics. The results described here suggest that the efficiency of structural controllability, previously linked to executive function [44, is positively correlated with neural dynamics that center the present. Future work could build upon a link between executive function and the regulation of emotions [73] to understand the relationship between attentional states of flow-defined as being effortlessly and fully involved in the present moment - when executing goal-directed tasks and well-being, mood, and self-regulation 74].

Our analyses of the neural dynamics of meditative practices suggests that future studies could shift their focus away from regional activity and toward network dynamics to provide additional insight into other neural processes of meditative practices in service of understanding the individual differences of their efficacy. In addition to effortful expenditure of control input to deautomatize and discontinue the processing of reactions to stimuli, other components beyond deautomatization remain relevant avenues of future study. Two such promising components are socially oriented regulatory strategies and cognitive flexibility. First, social influences serve an important role in self-regulatory processes, such as moderation of alcohol consumption 3]. Future research could study how socially oriented mindsets, such as loving-kindness meditation and perspective-taking, elicit mindsets characterized by care for others' well-being and openness to learning new information [69, 75]. Prior hypotheses suggest that social processing is an efficient baseline for self-regulation due to cooperation, shared resources, risk mitigation, and common goals [76]. Our findings naturally lead to the hypothesis that socially oriented mindsets may prime more efficient neural dynamics that bias neural dynamics to center recently experienced past messages that linger into the future. Indeed, average controllability is greatest in default mode network regions whose activity is commonly associated with social processes and thinking about the past or future [39]. Second, cognitive flexibility has been hypothesized to be a key component of how mindfulness supports self-regulation 24]. Prior work has shown that the time spent in a state, the frequency of state occurrence, and transitions among brain states are associated with flexibility 77] and learning [78. These approaches are well suited for research on the neural dynamics of meditative practices because dynamics between the default mode and frontoparietal networks have been associated with cognitive flexibility. As a major aim of mindfulness is non-elaborative and non-narrative processing of past experience, future work could apply event segmentation to assess how the narrative structure of resting-state dynamics becomes more or less elaborated in response to meditative practices and messaging [79, 80]. Understanding the neural processes by which meditative practices enhance self-regulation could inform models of health behavior change, addiction, and implicit bias that theorize a critical role for executive function to 
change automatic or habitual behavior; such models are crucial for the design of individualized interventions that support self-regulatory goals $[81,8,82]$.

Methodological Considerations. These findings should be considered within the context of the strengths and limitations of our approach. Strengths of the study included randomized intervention experiments, follow-up longitudinal measurements of the self-regulation of real-world behavior, multi-modal analyses using diffusion, task-based, and resting-state neuroimaging, and statistical modeling tailored to the non-normal distribution of drinking behavior beyond baseline drinking and personality differences. Moreover, network control theory was well positioned to overcome limitations of the theoretical definition of neural effort and efficiency that have prevented previous research from drawing conclusive inferences about the role of neural dynamics in meditative practices. Our use of the intrinsic timescale was also notable for its relevance to the definition of mindfulness [10], and for the fact that it provides empirical justification for the temporal stability that is typically inferred from the theoretical assumptions of control stability [66, 83, 34]. Limitations of the study included the simplifying assumption of linear neural dynamics, though this simplification is common and justifiable in macroscale networks [84, 85]. Another limitation was the use of an experimental benchmark task that was not expected to provide similar benefits as the interventions [14. While our primary aim was to understand the relations of neural effort, efficiency, and stability to mindful states, incorporating an active comparator experimental design will be crucial for studies of mindfulness interventions in clinical or educational settings. Moreover, other features of a neural signal can produce shorter intrinsic timescales, such as greater power of higher frequency oscillations. Other contributions of the signal to the stability of neural dynamics remain important avenues of investigation, preferably using neuroimaging methods like EEG with better temporal resolution.

Conclusion. Taken together, we described evidence that mindfulness was associated with effortful neural states with discontinuing neural dynamics. More effortful neural states tend to more quickly update and discontinue the past in order to center the present experience. Our results provide support for the potential use of various meditative practices to differently maximize the functional value and cost of thinking about the past, planning for the future, and staying in the present according to personal goals and contextual demands [21, 155, 71]. 


\section{Methods}

We used data from the Social and Health Impact of Network Effects (SHINE) study, a larger study designed to provide insight into health behaviors and social interactions among young adults. All research was conducted in accordance with the Institutional Review Board (IRB) at the University of Pennsylvania and Columbia University, in addition to the Army Research Office.

\section{Participants}

Participants were undergraduate students recruited from social groups (e.g., sororities, sports clubs, performance groups) at the University of Pennsylvania and Columbia University. Eligible social groups included on-campus organizations containing 20-100 members, with at least $80 \%$ of the members interested in participating in the study. Of eligible social groups, 925 individuals were invited to enroll in the study. These individuals were from 24 social groups across the two universities (33\% performing arts groups, $29 \%$ sororities or fraternities, $25 \%$ sports clubs, $8 \%$ technology clubs). Interested participants ( $N=583 ; 63 \%$ of invited participants) consented to participate and completed an hour-long baseline survey, as described below.

\section{Eligibility criteria}

Participants were eligible to enroll in the study if they were a member of one of the social groups invited to participate. Those who were willing to participate were invited to complete the baseline survey. Eligibility for the MRI session was determined by participant responses to questions in the baseline survey and the response completion rate of the social group. Social groups were eligible if more than 15 people completed the survey or if more than $20 \%$ of the group members completed the survey. Based on these criteria, 19 social groups were eligible. Of these groups, individuals were eligible to complete the MRI session if they: were 18 years or older, fluent in English, and free from MRI contraindications; weighed less than 350 lbs; were not claustrophobic, pregnant, or studying abroad at the time; had no history of serious medical issues, psychiatric hospitalization, or drug abuse; and drank alcohol and listed at least two people in their social group who drank the least in the group apart from themselves. Of the participants who completed the baseline survey 113 participants were invited to complete the MRI session. 


\section{Mindfulness intervention assignment}

Participants who enrolled in the MRI session component were randomly assigned to one of three intervention groups: mindfulness, perspective-taking, or benchmark. In the mindfulness and perspective-taking groups, participants were trained to use cognitive strategies to regulate their responses to alcohol cues. The benchmark participants was instructed to respond naturally without trying to alter their responses. In this paper, we focused on investigating the effects of mindfulness compared to the baseline condition.

\section{MRI acquisition, preprocessing, and modeling}

\section{Cue-reactivity fMRI task}

Consistent with past work on regulation of alcohol craving [3], we used images of alcohol (beer, wine, and liquor) to elicit craving. Before the task, participants were randomized to one of three groups (mindfulness, perspective-taking, or control) and were trained how to do the task based on their group. During the task, participants saw images of alcoholic (e.g., bottle of beer) and control images of non-alcoholic beverages (e.g., water bottle) selected from the Galician Beverage Picture Set 62. This normalized stimulus set contains images that are compositionally similar to one another and without beverage brands, and balances social contexts (e.g., alone versus in a social setting). While viewing the images, participants were either instructed to react naturally ("React" trials) or regulate their responses to the images ("Regulate" trials). After each image, they rated their craving on a 5 -point scale $(1=$ not at all, $5=$ very much $)$. On half of the React trials, participants saw images of alcoholic beverages; on the other half, they saw control, non-alcoholic beverages. Participants in the control group completed the React trials only, whereas participants in the mindfulness and perspective-taking groups completed both React and Regulate trials. On Regulate trials, participants in the mindfulness group were instructed to attend mindfully to their experience, accepting their thoughts and feelings in a non-judgemental way. Detailed instructions for the task are available at http://osf.io/gkahy/.

Participants completed 96 trials across 4 task runs. This task used a mixed-design in which trials were blocked per condition to reduce the burden associated with task-switching. Each block consisted of 4 trials and each task run consisted of 6 blocks. Each block began with a condition cue (3 seconds) followed by 4 trials, each consisting of an image presentation (6 seconds) and a craving rating (3 seconds); each event was separated by a jittered fixation cross $(\mathrm{M}=4.0$ seconds, $\mathrm{SD}=2.6$ seconds $)$. Block order was counterbalanced across participants within each group. Stimuli were presented using PsychoPy [86] and participants responded to 
stimuli using a five-button box. After the scan session, participants answered questions about the cognitive strategies they used during the task and their level of confidence using the strategies in the post-scan survey.

\section{MRI data acquisition}

Of the 113 participants who were eligible and enrolled for this component, 112 completed an MRI session at the University of Pennsylvania or Columbia University. During this session, participants completed a pre-scan survey, a 90-minute MRI scan that included structural, diffusion-weighted, resting-state, and task functional MRI (fMRI) scans, and a post-scan survey related to the fMRI tasks. Participants were also prepared to complete the experience sampling component. One participant was deemed ineligible for the MRI scan due to a contraindication discovered at the session, but they completed all behavioral components of the session. Another participant was scanned but the data was lost due to a technical error. This yielded a total of 77 participants in the mindfulness $(n=38)$ or benchmark $(n=39)$ groups.

Scans were acquired using 3-Tesla Siemens Prisma scanners equipped with a 64-channel head coil. For each participant, scans were acquired in the following sequence: a resting-state scan, two runs of a face perception ("faces") functional MRI (fMRI) task, a T1-weighted structural scan, four runs of a cue-reactivity fMRI task, a fieldmap for the diffusion-weighted (DWI) scan, a diffusion-weighted (DWI) scan, and a T2weighted structural scan. DICOM images were converted to NIfTI files in the Brain Imaging Data Structure 87. format using HeuDiConv [88. The DWI data were preprocessed and reconstructed through QSIprep (Version 0.8.0; [89]). Briefly, the data was first denoised and bias corrected, and then underwent susceptibility distortion correction, as well as motion and eddy current correction via FSL 6.0, and were coregistered to the T1 space. We also warped both the Schaefer atlas [90] and the Harvard Oxford subcortical atlas 91 into individual T1 space to subdivide the brain into 400 cortical and 14 subcortical regions. Then, the preprocessed DWI data was reconstructed using generalized $Q$-sampling Imaging [92] in DSI-Studio (http://dsi-studio.labsolver.org). Deterministic tractography [93] was performed until $5 \times 106$ streamlines were reconstructed, yielding individual structural networks with brain regions as nodes and the number of streamlines connecting each brain region pair as weighted edges. Preprocessing was performed using QSIPrep (Version 0.8.0), which is based on Nipype (Version 1.4.2; 94]). 


\section{Anatomical data preprocessing}

The structural, resting-state, and task-based fMRI scans were preprocessed using fMRIPrep (Version 20.0.6; 95]), which is based on Nipype (Version 1.4.2; 94]). The T1-weighted (T1w) image was corrected for intensity non-uniformity (INU) with N4BiasFieldCorrection [96, distributed with ANTs 2.2.0 [97, and used as T1w-reference throughout the workflow. The T1w-reference was then skull-stripped with a Nipype implementation of the ANTs brain extraction workflow, using OASIS30ANTs as the target template. Brain tissue segmentation of cerebrospinal fluid (CSF), white matter (WM) and gray matter (GM) was performed on the brain-extracted T1w using fast (FSL, Version 5.0.9; 98]). Brain surfaces were reconstructed using recon-all (FreeSurfer, Version 6.0.1; 99), and the brain mask estimated previously was refined with a custom variation of the method to reconcile ANTs-derived and FreeSurfer-derived segmentations of the cortical gray matter of Mindboggle [100]. Volume-based spatial normalization to one standard space (MNI152NLin2009cAsym; 101]) was performed through nonlinear registration with antsRegistration (ANTs, Version 2.2.0), using brain-extracted versions of both the T1w reference and the T1w template.

\section{Resting-state and task-fMRI data preprocessing}

For each of the resting-state and task BOLD scans, the following preprocessing was performed. First, a reference volume and its skull-stripped version were generated using a custom methodology of fMRIPrep. A B0-nonuniformity map (or fieldmap) was estimated based on two echo-planar imaging (EPI) references with opposing phase-encoding directions, with 3dQwarp in AFNI [102]. Based on the estimated susceptibility distortion, a corrected EPI reference was calculated for a more accurate co-registration with the anatomical reference. The BOLD reference was then co-registered to the T1w reference using bbregister from FreeSurfer, which implements boundary-based registration [103. Co-registration was configured with six degrees of freedom. Head-motion parameters with respect to the BOLD reference (transformation matrices, and six corresponding rotation and translation parameters) are estimated before any spatiotemporal filtering using mcflirt (FSL, Version 5.0.9; [104]). BOLD runs were slice-time corrected using 3dTshift from AFNI. The BOLD time-series were resampled onto their original, native space by applying a single, composite transform to correct for head motion and susceptibility distortions. The BOLD time-series were resampled into standard space, generating a preprocessed BOLD run in MNI152NLin2009cAsym space. All resamplings were performed with a single interpolation step by composing all the pertinent transformations (i.e. head-motion transform matrices, susceptibility distortion correction when available, and co-registrations to anatomical and output spaces). Gridded (volumetric) resamplings were performed using antsApplyTransforms (ANTs), 
configured with Lanczos interpolation to minimize the smoothing effects of other kernels. Non-gridded (surface) resamplings were performed using mri_vol2surf (FreeSurfer). Various confounds (e.g., framewise displacement, DVARS, global signal) were also calculated for each TR. The outputs from fMRIPrep were then manually quality checked to ensure adequate preprocessing.

\section{fMRI motion regression}

Following preprocessing with fMRIPrep, these data were denoised using the xcpEngine pipeline [105. Specifically, xcpEngine was used to remove motion-related confounds from BOLD sequences using the most stringent of current standards [105]. These steps were as follows: (1) demeaning and removal of linear and quadratic trends from time series, (2) de-spiking using AFNI's 3DDESPIKE utility, (3) temporal bandpass filtering using a first-order Butterworth filter to retain signal in the range $0.01-0.08 \mathrm{~Hz}$, (4) 36-parameter confound regression including 6 realignment parameters, mean signal in white matter, CSF and mean global signal, as well as the first power and quadratic expansions of their temporal derivatives. These denoised time series were then used in further analyses.

Prior to first-level modeling, we generated motion regressors using an automated motion assessment tool (106; ; https://github.com/dcosme/auto-motion-fmriprep). This tool is a predictive model that utilizes the confound files generated by fMRIPrep and classifies whether or not fMRI volumes contain motion artifacts. The classifier is applied to each participant's task run and returns a binary classification indicating the presence or absence of motion artifacts for each volume. In addition, this tool transforms the realignment parameters into Euclidean distance for translation and rotation separately, and calculates the displacement derivative of each. This procedure yields a total of 5 motion regressors for first-level modeling. Task runs that contain $>10 \%$ of volumes classified as containing a motion artifact will be excluded from further analyses $(n=1)$. For group-level analyses, multiple comparisons will be corrected using cluster-extent thresholding was implemented using AFNI [102]. In accordance with recent guidelines [107, the spatial autocorrelation function was first estimated for each subject and task run separately using AFNI's 3dFWHMx on the residuals, and then averaged across subjects. To determine probability estimates of false-positive clusters given a random field of noise, Monte-Carlo simulations were conducted with AFNI's 3dClustSim using the average autocorrelation across subjects. Following motion exclusions, the sample included $n=37$ mindfulness and $n=39$ benchmark participants. 


\section{Diffusion data preprocessing}

MP-PCA denoising as implemented in MRtrix3's dwidenoise [108] was applied with a 5-voxel window. After MP-PCA, Gibbs unringing was performed using MRtrix3's mrdegibbs [109. Following unringing, B1 field inhomogeneity was corrected using dwibiascorrect from MRtrix3 with the N4 algorithm [96. After B1 bias correction, the mean intensity of the DWI series was adjusted so that the mean intensity of the $b=0$ images was matched across DWI scanning sequences. FSL (Version 6.0.3:b862cdd5)'s eddy was used for head motion correction and eddy current correction [110. Eddy was configured with a $q$-space smoothing factor of 10, a total of 5 iterations, and 1000 voxels used to estimate hyperparameters. A linear first level model and a linear second level model were used to characterize spatial distortions related to eddy currents. The $q$-space coordinates were forcefully assigned to shells. The field offset was attempted to be separated from subject movement. Shells were aligned after eddy current correction. Eddy's outlier replacement was run [110. Data were grouped by slice, only including values from slices determined to contain at least 250 intracerebral voxels. Groups deviating by more than 4 standard deviations from the prediction had their data replaced with imputed values. Fieldmaps were collected with reversed phase-encode blips, resulting in pairs of images with distortions going in opposite directions. Here, a $b=0$ fieldmap image with reversed phase encoding direction was used along with $b=0$ images extracted from the DWI scans. From these pairs, the susceptibility-induced off-resonance field was estimated using a method similar to that described in Ref. [111. The fieldmaps were ultimately incorporated into the eddy current and head motion correction interpolation. Final interpolation was performed using the jac method.

Several confounding time-series were calculated based on the preprocessed DWI. Framewise displacement (FD) was calculated using the implementation in Nipype (following the definitions in [112]). The headmotion estimates calculated in the correction step were also placed within the corresponding confounds file. Slicewise cross correlation was also calculated. The DWI time-series were resampled to ACPC, generating a preprocessed DWI run in ACPC space with $1.7 \mathrm{~mm}$ isotropic voxels. Many internal operations of QSIPrep use Nilearn (Version 0.7.0; [113]) and Dipy [114. 


\section{Ecological momentary assessments and mindfulness interventions}

\section{Ecological momentary assessment}

After completing the MRI session, participants $(N=108)$ began a 28-day experience sampling assessment that measured daily drinking behavior, mood, craving, and emotion regulation, among other factors. For participants in the mindfulness and benchmark groups, the experience sampling procedure also served as an intervention by reminding the participants of the instructions for how to regulate their responses to alcohol. These interventions reminded participants to employ the cognitive strategies that they learned during training in the cue-reactivity task while undergoing fMRI, as well as in the 28-day experience sampling component. The mindfulness intervention was delivered on alternating weeks during the experience sampling component. On these "on" weeks, participants received two prompts a day (at 2PM and 9PM) reminding them to use the cognitive strategy when they encountered alcohol. On "off" weeks, participants were instructed to react naturally to alcohol cues ("If you are around alcohol today, REACT NATURALLY — have whatever thoughts and feelings you would normally have."). This approach was adopted in order to assess within-person effects of the intervention; intervention delivery week order (on/off/on/off or off/on/off/on) was counterbalanced across participants.

\section{Mindfulness intervention}

The mindfulness intervention was based on instructions that were iteratively refined across 14 pilot studies conducted online via Amazon's Mechanical Turk. Participants in the mindfulness group were trained to approach alcohol cues mindfully, "by mentally taking a step back in order to observe the situation and

[their] responses in an impartial and non-judgmental manner." They were also trained to pay attention to their reactions without getting caught up in them. On intervention ("on") weeks in the experience sampling component, participants were reminded to respond mindfully to alcohol cues twice a day ("If you are around alcohol today, REACT MINDFULLY — notice, acknowledge, and accept the thoughts and feelings you have.").

Participants in the control group were not trained to use any cognitive strategy to change their responses to alcohol. Instead, they were instructed to approach alcohol cues naturally, without regulating their responses ("If you are around alcohol today, REACT NATURALLY - have whatever thoughts and feelings you would 
normally have.") throughout the whole sampling period.

\section{Ecological assessment measure of drinking}

Drinking was defined as the number of alcohol servings consumed per assessment distributed throughout the 28-day period. To obtain the number of alcohol servings per occasion, we summed the number of wine, beer, and liquor drinks at each signal level. The three largest and improbable values of drinks per drinking occasion $(24,36,60)$ observed across three individuals, were set to 16 drinks per occasion. We observed no substantial differences in results when including these outlier values in the main models as part of additional sensitivity analyses.

\section{Network control theory}

\section{Average controllability}

From the DWI data, we constructed anatomical brain networks by subdividing the brain into 414 regions using the Schaefer atlas for 400 cortical regions and the Harvard Oxford atlas for 14 subcortical regions 90, 91, 115. In these anatomical connectivity matrices, brain regions are defined as nodes, and a link between two nodes represents the number of streamlines connecting them. Controllability of a dynamical system describes the possibility of driving the current state of a system to a desired target state via external control input [38]. Such an approach allows us to gain better insight into the relationship between brain structure and brain dynamics. Here, we focus on average controllability, which quantifies each region's capacity to leverage the brain's underlying structural connectivity to distribute activity throughout the brain to guide changes to any new hypothetical states [39. Networks with high average controllability are more influential in the control of network dynamics than networks with low average controllability. The relationship between the mathematical formulation of network control and brain networks is discussed in more detail elsewhere [116]. To ensure system stability, each participant's structural connectivity matrix was normalized by dividing each element by the largest absolute eigenvalue of the matrix plus one [38. Following normalization, average controllability was calculated for each node. Finally, we calculated the mean average controllability over nodes. These person-averaged estimates of average controllability were then considered further in the subsequent analyses of between-person differences. 


\section{Optimal control inputs and control stability}

We applied network control theory metrics to investigate the effect of mindfulness in driving brain state trajectories associated with processing alcohol cues. The network control theory framework has been used to determine how underlying white matter architecture constrains transitions between different brain states inferred from neuroimaging data [34, 35, 36, 37]. The control inputs required to execute these transitions between brain states can be thought of as a way of operationalizing cognitive effort, following prior work [39, 34. We begin by approximating brain state dynamics through the linear continuous-time equation

$$
\dot{x}(t)=A x(t)+B u(t),
$$

where, for the task fMRI data, $x(t)$ is a vector of size $N \times 1$ (where $N$ is the 414 brain regions in the network, consisting of 400 cortical regions and 14 subcortical regions) that represents the state of the system at time $t, A$ is the weighted symmetric $N \times N$ structural matrix estimated through diffusion spectrum imaging, $B$ is an input matrix of size $N \times N$ specifying the set of control nodes, and $u(t)$ is the time-dependent control signal in each of the control nodes. When analyzing the resting-state fMRI data, $x(t)$ is defined as a $N$ x 1 vector from the the $N \times R$ BOLD time series, where $R$ is the set of 300 images acquired at each repetition time during the scan. The optimal control energy framework 38 defines the unique control input $u^{*}(t)$ needed to transition the system from an initial state $x(0)=x_{0}$ to a final target state $x(T)=x_{T}$ over the time horizon $T$ through the cost function that solves the problem:

$$
\begin{aligned}
& \boldsymbol{u}(t)_{\kappa}^{*}=\underset{u_{K}}{\operatorname{argmin}} J\left(\boldsymbol{u}_{\kappa}\right)=\underset{\boldsymbol{u}_{\kappa}}{\operatorname{argmin}} \int_{0}^{T} \\
& \left(\left(\boldsymbol{x}_{T}-\boldsymbol{x}(t)\right)^{\top}\left(\boldsymbol{x}_{T}-\boldsymbol{x}(t)\right)+\rho \boldsymbol{u}_{\kappa}(t)^{\top} \boldsymbol{u}_{\kappa}(t)\right) d t,
\end{aligned}
$$

where the parameter $\rho$ determines the relative weighting between the costs associated with the length of the state trajectory and the input energy. Following prior work, we set $\delta$ to 1 such that no specific assumptions are made about the relative importance of constraints on energy and distance values 38 . The cost function $J\left(\boldsymbol{u}(t)_{\kappa}^{*}\right)$ is defined to find the unique optimal control input $\boldsymbol{u}(t)_{\kappa}^{*}$. We then use this optimal control input to calculate the control inputs required by a single brain region:

$$
E_{i}^{*}=\int_{0}^{T}\left\|u_{i}^{*}(t)\right\|_{2}^{2} d t .
$$

By integrating each control input over time, we can calculate the total control energy required by all brain regions: 


$$
E^{*}=\int_{0}^{T} u(t)^{\mathrm{T}} u(t) d t
$$

In our model, we used parameter estimates ( $\beta$ weights from a general linear model) to specify brain states from the cue-reactivity task. The baseline state was defined by $\beta$ weights equal to 0 (no relationship between neural activity and task). Each participant's structural matrix was used as $A$. We defined $B$ to allow control inputs into the 145 brain regions of the dorsal attention, ventral attention, and frontoparietal networks, following prior cognitive neuroscience literature implicating these networks in exerting executive control resources [21, 39, 90]. Following prior work showing that the choice of $T$ results in high correlated (Pearson's $r>0.99$ ) control input values across different values of $T$, we chose $T=3[117$.

We sought to model the control inputs required to sustain the same neural state, which allows us to determine the control stability. The persistence energy is the control input $E_{i}^{*}$ required to transition from $x_{T}$ to $x_{T}$. With the terminology above, persistence energy defines $x_{0}=x_{T}$. Assuming that states which require greater persistence energy are less stable, the control stability $S$ of a neural state is defined as

$$
S=\frac{1}{\log _{10}\left(E_{x_{0}=x_{T}}\right)} .
$$

While the control input operationalizes cognitive effort of neural dynamics, the control stability operationalizes the temporal stability of neural states.

\section{Intrinsic timescale}

We sought to also investigate the stability of neural states using existing metrics that estimate the similarity of neural states over time from the regional time course data. To determine the temporal stability and similarity of neural states, we calculated the intrinsic timescale per brain region using the denoised time series after removal of motion confounds 105 . The intrinsic timescale operationalizes the temporal windows by which regional computations are thought to unfold [50. These temporal windows may be arranged hierarchically such that the computational timescales increase as activity propagates from unimodal sensorimotor to transmodal frontoparietal, default mode, and limibic cortices [118. To determine the intrinsic timescale, we used a model-free estimate from a previously published method [51]. Briefly, the method determines the duration required for a signal to decay by half, and does so by estimating the full width at half maximum of an autocorrelation function. We used this previously published method and corresponding MATLAB code 
available at https://github.com/ryraut/intrinsic-timescales

\section{Statistical modeling}

To examine associations between mindfulness and average controllability on drinking throughout the 28day EMA protocol, we used multilevel models. The multilevel models accommodated the nested nature of the smartphone sampling data including 56 total alcohol conversation signals and 56 total drinking signals, nested in 104 participants across 10 social groups. We chose a multilevel hurdle model 64] using glmmTMB in R, specifying a truncated negative binomial function [119]. Count data such as alcohol use data in the present case (i.e., number of drinks consumed) are often positively skewed and include many observations at zero. Hurdle models are often used to model this type of data as these include both a count regression (in this case Poisson/negative binomial) to model the counts, and a logistic regression to model the zeroes in the data. Here, all the counts are modeled by a truncated Poisson/negative binomial (i.e., truncated as it does not contain zero), and all the zeroes (non-alcohol use occasions) are modeled with the logistic regression. Thus, using these models allowed us to independently model and isolate how much a person drinks when they drink, our primary outcome, and to model the probability of whether a person drinks or not at a given occasion as part of secondary analyses. We specified nested random effects to include participant ID and group ID, given that the repeated conversational valence and drinking observations are nested within participants over 28 days, and those participants in turn are nested within 10 social groups.

Recent work used the most parsimonious models of alcohol consumption behavior change by controlling for a number of covariates described below (manuscript in preparation). First, to isolate occasions when participants talked more positively about alcohol, relative to their own baseline and prior to a drinking occasion at the within-person level, we controlled for each person's usual month level between-person alcohol conversation valence over the 28 days. We additionally controlled for time in the study, as individuals reported drinking less towards the end of the ecological momentary assessment period; social weekend (Thursday, Friday, or Saturday vs. weekday), given different drinking patterns on "college weekends" vs. weekdays among students [65]; and smartphone survey response rates. We additionally controlled for demographic variables such as gender, as drinking patterns often vary across gender [120]; age, the number of alcohol conversations, and randomization into a drinking intervention as part of a different study. Sensitivity analyses were conducted to test for the robustness and overfitting of subsets of these models (manuscript in preparation). 
In this work, we were additionally interested in assessing how average controllability influences later drinking. Following prior work studying the effects of loving-kindness meditation and self-affirmation on self-regulation and behavior change [69], we controlled for additional covariates of baseline drinking, race/ethnicity, and years of education. Including baseline measurements for the frequency or amount of drinking alters the primary outcome model to be the residualized change from drinking at baseline to drinking during the mindfulness interventions. Lastly, we controlled for the personality trait of attentional impulsivity for four reasons. First, prior work suggests that self-reported mindfulness is negatively correlated with self-reported impulsivity 11. Second, prior work suggests that controlling for baseline personality differences could strengthen analytic designs testing for the effects of mindfulness [14. Third, despite differences of and debate regarding the definition of mindfulness as used by practioners, clinicians, and psychologists, the regulation of attention is a shared component [19]. Fourth and finally, mindfulness meditation has been shown to facilitate attention regulation and emotion regulation, supported by a relationship between self-reported levels of mindfulness and self-reported measures of adaptive emotion regulation strategies, even after controlling for symptoms of stress, anxiety, and depression [121. Attention impulsivity was positively correlated with difficulties in emotion regulation in our dataset and was not collinear with average controllability (Supplementary Figure 2).

The final models are the zero-inflated model of logistic drinking probability as a binary outcome and the conditional model of the count of drinking amount. They can be written as

Drinking probability $\sim b_{0}+b_{1}$ (Condition) $+b_{2}$ (Time) $+b_{3}$ (Gender $)+b_{4}($ Number of responses $)+$ $b_{5}($ Social Weekend $)+b_{6}($ Baseline drinking frequency $)+b_{7}($ Age $)+b_{8}($ Intervention week $)+$ $b_{9}($ Average controllability $)+b_{10}($ Race/ethnicity $)+b_{11}($ Years of education $)+b_{12}($ Attentional impulsivity $)+$ (1|group/participant).

Drinking amount $\sim b_{0}+b_{1}$ (Condition) $+b_{2}$ (Time) $+b_{3}$ (Gender $)+b_{4}($ Number of responses $)+$ $b_{5}($ Social Weekend $)+b_{6}($ Baseline drinking amount $)+b_{7}($ Age $)+b_{8}($ Intervention week $)+$ $b_{9}($ Average controllability $)+b_{10}($ Race/ethnicity $)+b_{11}($ Years of education $)+b_{12}($ Attentional impulsivity $)+$ (1)group/participant).

The predicted probability of drinking was plotted by using the regression model to generate predicted values of the marginal effect of average controllability. 


\section{Code availability}

The code for the analyses of this paper are available at https://github.com/dalejn/contemplativeControl.

\section{Data availability}

All data used in the manuscript are available at http://osf.io/gkahy/.

\section{Citation Diversity Statement}

Recent work in several fields of science has identified a bias in citation practices such that papers from women and other minority scholars are under-cited relative to the number of such papers in the field [122, 123, 124, 125, 126, 127, 128, 129, 130. Here we sought to proactively consider choosing references that reflect the diversity of the field in thought, form of contribution, gender, race, ethnicity, and other factors. First, we obtained the predicted gender of the first and last author of each reference by using databases that store the probability of a first name being carried by a woman [126, 131. By this measure (and excluding self-citations to the first and last authors of our current paper), our references contain $17.64 \%$ woman(first)/woman(last), $11.7 \% \mathrm{man} /$ woman, $22.9 \%$ woman/man, and $47.76 \% \mathrm{man} / \mathrm{man}$. This method is limited in that a) names, pronouns, and social media profiles used to construct the databases may not, in every case, be indicative of gender identity and b) it cannot account for intersex, non-binary, or transgender people. Second, we obtained predicted racial/ethnic category of the first and last author of each reference by databases that store the probability of a first and last name being carried by an author of color 132, 133. By this measure (and excluding self-citations), our references contain $9.7 \%$ author of color (first)/author of color(last), $10.8 \%$ white author/author of color, $23.07 \%$ author of color/white author, and $56.43 \%$ white author/white author. This method is limited in that a) names and Florida Voter Data to make the predictions may not be indicative of racial/ethnic identity, and b) it cannot account for Indigenous and mixed-race authors, or those who may face differential biases due to the ambiguous racialization or ethnicization of their names. We look forward to future work that could help us to better understand how to support equitable practices in science.

\section{Acknowledgements}

Research was sponsored by the Army Research Office and was accomplished under Grant Number W911NF18-1-0244. D.Z. acknowledges support from the National Institute of Mental Health (F31MH126569). D.M.L. 
acknowledges support from the National Institute on Drug Abuse (K01 DA047417) and the Brain \& Behavior Research Foundation. D.S.B. acknowledges support from the John D. and Catherine T. MacArthur Foundation, the Swartz Foundation, the Paul G. Allen Family Foundation, the Alfred P. Sloan Foundation and the NSF (PHY-1554488; IIS-1926757). D.S.B. and L. P. acknowledge support from the National Institute of Mental Health (R01MH113550). L.P. was also supported by the National Institute Of Mental Health (K99MH127296) and a 2020 NARSAD Young Investigator Grant from the Brain \& Behavior Research Foundation. The content is solely the responsibility of the authors and does not necessarily represent the official views of the National Institutes of Health. The views and conclusions contained in this document are those of the authors and should not be interpreted as representing the official policies, either expressed or implied, of the Army Research Office or the U.S. Government. The U.S. Government is authorized to reproduce and distribute reprints for Government purposes notwithstanding any copyright notation herein.

\section{Author contributions}

D.Z wrote the paper. Y.K., D.C., M.J., A.M., J.K.B., E.C., L.P., P.J.M, D.M.L., E.B.F. and D.S.B. edited the paper. D.Z. designed the analysis with input from D.S.B. Y.K., D.C., X.H., A.M., M.J., and N.C. preprocessed the data. D.Z. performed the analysis with input from D.C., A.M., X.H., M.J., N.C., D.M.L., J.K.B., E.B.F., and D.S.B. E.B.F., P.J.M., K.N.O., D.M.L., and D.S.B. acquired funding for data collection and designed the overarching study.

\section{Competing interests}

The authors declare that they have no competing interests.

\section{References}

[1] C. S. Carver and M. F. Scheier, On the self-regulation of behavior. Cambridge University Press, 2001.

[2] J. T. Hustad, K. B. Carey, M. P. Carey, and S. A. Maisto, "Self-regulation, alcohol consumption, and consequences in college student heavy drinkers: A simultaneous latent growth analysis," Journal of studies on alcohol and drugs, vol. 70, no. 3, pp. 373-382, 2009. 
[3] N. H. Naqvi, K. N. Ochsner, H. Kober, A. Kuerbis, T. Feng, M. Wall, and J. Morgenstern, "Cognitive regulation of craving in alcohol-dependent and social drinkers," Alcoholism: Clinical and Experimental Research, vol. 39, no. 2, pp. 343-349, 2015.

[4] S. G. Hofmann, P. Grossman, and D. E. Hinton, "Loving-kindness and compassion meditation: Potential for psychological interventions," Clinical psychology review, vol. 31, no. 7, pp. 1126-1132, 2011.

[5] K. Rubia, "The neurobiology of meditation and its clinical effectiveness in psychiatric disorders," Biological psychology, vol. 82, no. 1, pp. 1-11, 2009.

[6] C. J. Armitage, P. R. Harris, and M. A. Arden, "Evidence that self-affirmation reduces alcohol consumption: randomized exploratory trial with a new, brief means of self-affirming.," Health Psychology, vol. 30 , no. 5 , p. $633,2011$.

[7] J. L. Howell and J. A. Shepperd, "Reducing information avoidance through affirmation," Psychological science, vol. 23, no. 2, pp. 141-145, 2012.

[8] Y. Kang, J. R. Gray, and J. F. Dovidio, "The nondiscriminating heart: lovingkindness meditation training decreases implicit intergroup bias.," Journal of Experimental Psychology: General, vol. 143, no. 3, p. 1306, 2014.

[9] M. B. MacKenzie and N. L. Kocovski, "Mindfulness-based cognitive therapy for depression: trends and developments.," Psychology research and behavior management, 2016.

[10] N. Thera, "The power of mindfulness," Wheel Publication, pp. 1-75, 1968.

[11] K. W. Brown and R. M. Ryan, "The benefits of being present: mindfulness and its role in psychological well-being.," Journal of personality and social psychology, vol. 84, no. 4, p. 822, 2003.

[12] E. M. Seppala, C. A. Hutcherson, D. T. Nguyen, J. R. Doty, and J. J. Gross, "Loving-kindness meditation: A tool to improve healthcare provider compassion, resilience, and patient care," Journal of Compassionate Health Care, vol. 1, no. 1, pp. 1-9, 2014.

[13] A. M. Sweeney and A. Moyer, "Self-affirmation and responses to health messages: a meta-analysis on intentions and behavior.," Health Psychology, vol. 34, no. 2, p. 149, 2015.

[14] Y.-Y. Tang, B. K. Hölzel, and M. I. Posner, "The neuroscience of mindfulness meditation," Nature Reviews Neuroscience, vol. 16, no. 4, pp. 213-225, 2015. 
[15] L. M. McTeague, M. S. Goodkind, and A. Etkin, "Transdiagnostic impairment of cognitive control in mental illness," Journal of psychiatric research, vol. 83, pp. 37-46, 2016.

[16] S. G. Hofmann and G. J. Asmundson, "Acceptance and mindfulness-based therapy: New wave or old hat?," Clinical psychology review, vol. 28, no. 1, pp. 1-16, 2008.

[17] B. Luna, A. Padmanabhan, and K. O'Hearn, "What has fmri told us about the development of cognitive control through adolescence?," Brain and cognition, vol. 72, no. 1, pp. 101-113, 2010.

[18] S. Whitfield-Gabrieli and J. M. Ford, "Default mode network activity and connectivity in psychopathology," Annual Review of Clinical Psychology, vol. 8, no. 1, pp. 49-76, 2012.

[19] A. Chiesa, A. Serretti, and J. C. Jakobsen, "Mindfulness: Top-down or bottom-up emotion regulation strategy?," Clinical psychology review, vol. 33, no. 1, pp. 82-96, 2013.

[20] J. A. Brewer, P. D. Worhunsky, J. R. Gray, Y.-Y. Tang, J. Weber, and H. Kober, "Meditation experience is associated with differences in default mode network activity and connectivity," Proceedings of the National Academy of Sciences, vol. 108, no. 50, pp. 20254-20259, 2011.

[21] J. Smallwood and J. W. Schooler, "The restless mind.," Psychological bulletin, vol. 132, no. 6, p. 946, 2006.

[22] A. Lutz, H. A. Slagter, J. D. Dunne, and R. J. Davidson, "Attention regulation and monitoring in meditation," Trends in cognitive sciences, vol. 12, no. 4, pp. 163-169, 2008.

[23] R. Chambers, E. Gullone, and N. B. Allen, "Mindful emotion regulation: An integrative review," Clinical Psychology Review, vol. 29, no. 6, pp. 560-572, 2009.

[24] Y. Kang, J. Gruber, and J. R. Gray, "Deautomatization of cognitive and emotional life," The Wiley Blackwell handbook of mindfulness, vol. 1, p. 168, 2014.

[25] R. A. Poldrack, "Is "efficiency" a useful concept in cognitive neuroscience?," Developmental cognitive neuroscience, vol. 11, pp. 12-17, 2015.

[26] K. C. Fox, M. L. Dixon, S. Nijeboer, M. Girn, J. L. Floman, M. Lifshitz, M. Ellamil, P. Sedlmeier, and K. Christoff, "Functional neuroanatomy of meditation: A review and meta-analysis of 78 functional neuroimaging investigations," Neuroscience \&J Biobehavioral Reviews, vol. 65, pp. 208-228, 2016. 
[27] G. Buzsáki, K. Kaila, and M. Raichle, "Inhibition and brain work," Neuron, vol. 56, no. 5, pp. 771-783, 2007.

[28] R. Cools and M. D'Esposito, "Inverted-u-shaped dopamine actions on human working memory and cognitive control," Biological psychiatry, vol. 69, no. 12, pp. e113-e125, 2011.

[29] D. S. Bassett, P. Zurn, and J. I. Gold, "On the nature and use of models in network neuroscience," Nature Reviews Neuroscience, vol. 19, no. 9, pp. 566-578, 2018.

[30] A. E. Sizemore and D. S. Bassett, "Dynamic graph metrics: Tutorial, toolbox, and tale," NeuroImage, vol. 180, pp. 417-427, 2018.

[31] F. Pasqualetti, S. Zampieri, and F. Bullo, "Controllability metrics, limitations and algorithms for complex networks," IEEE Transactions on Control of Network Systems, vol. 1, no. 1, pp. 40-52, 2014.

[32] J. G. T. Zañudo, G. Yang, and R. Albert, "Structure-based control of complex networks with nonlinear dynamics," Proceedings of the National Academy of Sciences, vol. 114, no. 28, pp. 7234-7239, 2017.

[33] Z. Chen and S. V. Sarma, "Dynamic neuroscience," Chen and SV Sarma, Eds. Cham: Springer International Publishing AG, 2018.

[34] U. Braun, A. Harneit, G. Pergola, T. Menara, A. Schäfer, R. F. Betzel, Z. Zang, J. I. Schweiger, X. Zhang, K. Schwarz, et al., "Brain network dynamics during working memory are modulated by dopamine and diminished in schizophrenia," Nature Communications, vol. 12, no. 1, pp. 1-11, 2021.

[35] R. F. Betzel, S. Gu, J. D. Medaglia, F. Pasqualetti, and D. S. Bassett, "Optimally controlling the human connectome: the role of network topology," Scientific reports, vol. 6, no. 1, pp. 1-14, 2016.

[36] S. Gu, R. F. Betzel, M. G. Mattar, M. Cieslak, P. R. Delio, S. T. Grafton, F. Pasqualetti, and D. S. Bassett, "Optimal trajectories of brain state transitions," Neuroimage, vol. 148, pp. 305-317, 2017.

[37] J. Stiso, A. N. Khambhati, T. Menara, A. E. Kahn, J. M. Stein, S. R. Das, R. Gorniak, J. Tracy, B. Litt, K. A. Davis, et al., "White matter network architecture guides direct electrical stimulation through optimal state transitions," Cell reports, vol. 28, no. 10, pp. 2554-2566, 2019.

[38] T. M. Karrer, J. Z. Kim, J. Stiso, A. E. Kahn, F. Pasqualetti, U. Habel, and D. S. Bassett, "A practical guide to methodological considerations in the controllability of structural brain networks," Journal of neural engineering, vol. 17, no. 2, p. 026031, 2020. 
[39] S. Gu, F. Pasqualetti, M. Cieslak, Q. K. Telesford, B. Y. Alfred, A. E. Kahn, J. D. Medaglia, J. M. Vettel, M. B. Miller, S. T. Grafton, et al., "Controllability of structural brain networks," Nature communications, vol. 6, p. 8414, 2015.

[40] A. Shenhav, M. M. Botvinick, and J. D. Cohen, "The expected value of control: an integrative theory of anterior cingulate cortex function," Neuron, vol. 79, no. 2, pp. 217-240, 2013.

[41] R. Cools, "Chemistry of the adaptive mind: lessons from dopamine," Neuron, vol. 104, no. 1, pp. 113$131,2019$.

[42] E. Tang, C. Giusti, G. L. Baum, S. Gu, E. Pollock, A. E. Kahn, D. R. Roalf, T. M. Moore, K. Ruparel, R. C. Gur, et al., "Developmental increases in white matter network controllability support a growing diversity of brain dynamics," Nature communications, vol. 8, no. 1, pp. 1-16, 2017.

[43] W. H. Lee, A. Rodrigue, D. C. Glahn, D. S. Bassett, and S. Frangou, "Heritability and cognitive relevance of structural brain controllability," Cerebral Cortex, vol. 30, no. 5, pp. 3044-3054, 2020.

[44] E. J. Cornblath, E. Tang, G. L. Baum, T. M. Moore, A. Adebimpe, D. R. Roalf, R. C. Gur, R. E. Gur, F. Pasqualetti, T. D. Satterthwaite, and D. S. Bassett, "Sex differences in network controllability as a predictor of executive function in youth," NeuroImage, vol. 188, pp. 122-134, 2019.

[45] K. N. Ochsner and J. J. Gross, "The cognitive control of emotion," Trends in cognitive sciences, vol. 9, no. 5, pp. 242-249, 2005.

[46] W. M. Klein and P. R. Harris, "Self-affirmation enhances attentional bias toward threatening components of a persuasive message," Psychological Science, vol. 20, no. 12, pp. 1463-1467, 2009.

[47] A. L. McGowan, L. Parkes, X. He, O. Stanoi, Y. Kang, S. Lomax, P. Mucha, K. N. Ochsner, E. Falk, D. S. Bassett, et al., "Controllability of structural brain networks and the waxing and waning of negative affect in daily life," 2021.

[48] E. Bullmore and O. Sporns, "The economy of brain network organization," Nature Reviews Neuroscience, vol. 13, no. 5, pp. 336-349, 2012.

[49] D. Zhou, C. W. Lynn, Z. Cui, R. Ciric, G. L. Baum, T. M. Moore, D. R. Roalf, J. A. Detre, R. C. Gur, R. E. Gur, et al., "Efficient coding in the economics of human brain connectomics," arXiv preprint arXiv:2001.05078, 2020. 
[50] J. D. Murray, A. Bernacchia, D. J. Freedman, R. Romo, J. D. Wallis, X. Cai, C. Padoa-Schioppa, T. Pasternak, H. Seo, D. Lee, et al., "A hierarchy of intrinsic timescales across primate cortex," Nature neuroscience, vol. 17, no. 12, pp. 1661-1663, 2014.

[51] R. V. Raut, A. Z. Snyder, and M. E. Raichle, "Hierarchical dynamics as a macroscopic organizing principle of the human brain," Proceedings of the National Academy of Sciences, vol. 117, no. 34, pp. 20890-20897, 2020.

[52] W. S. Slutske, "Alcohol use disorders among us college students and their non-college-attending peers," Archives of general psychiatry, vol. 62, no. 3, pp. 321-327, 2005.

[53] C. A. Presley, P. W. Meilman, and R. Lyerla, "Development of the core alcohol and drug survey: Initial findings and future directions," Journal of American College Health, vol. 42, no. 6, pp. 248-255, 1994.

[54] K. Christoff, Z. C. Irving, K. C. Fox, R. N. Spreng, and J. R. Andrews-Hanna, "Mind-wandering as spontaneous thought: a dynamic framework," Nature Reviews Neuroscience, vol. 17, no. 11, pp. 718$731,2016$.

[55] K. D. Gerlach, R. N. Spreng, A. W. Gilmore, and D. L. Schacter, "Solving future problems: default network and executive activity associated with goal-directed mental simulations," Neuroimage, vol. 55, no. 4, pp. 1816-1824, 2011.

[56] M. H. Lee, C. D. Smyser, and J. S. Shimony, "Resting-state fmri: a review of methods and clinical applications," American Journal of neuroradiology, vol. 34, no. 10, pp. 1866-1872, 2013.

[57] S. Fiske, "Examining the role of intent: Toward understanding its role in stereotyping and prejudice.," 1989.

[58] S. Nolen-Hoeksema, "Responses to depression and their effects on the duration of depressive episodes.," Journal of abnormal psychology, vol. 100, no. 4, p. 569, 1991.

[59] P. R. Goldin, K. McRae, W. Ramel, and J. J. Gross, "The neural bases of emotion regulation: reappraisal and suppression of negative emotion," Biological psychiatry, vol. 63, no. 6, pp. 577-586, 2008.

[60] Z. V. Segal, M. Williams, and J. Teasdale, Mindfulness-based cognitive therapy for depression. Guilford Publications, 2018. 
[61] C. N. Cascio, M. B. O’Donnell, F. J. Tinney, M. D. Lieberman, S. E. Taylor, V. J. Strecher, and E. B. Falk, "Self-affirmation activates brain systems associated with self-related processing and reward and is reinforced by future orientation," Social cognitive and affective neuroscience, vol. 11, no. 4, pp. 621-629, 2016.

[62] E. López-Caneda and C. Carbia, "The galician beverage picture set (gbps): A standardized database of alcohol and non-alcohol images," Drug and alcohol dependence, vol. 184, pp. 42-47, 2018.

[63] M. D. Mrazek, J. Smallwood, and J. W. Schooler, "Mindfulness and mind-wandering: finding convergence through opposing constructs.," Emotion, vol. 12, no. 3, p. 442, 2012.

[64] D. C. Atkins, S. A. Baldwin, C. Zheng, R. J. Gallop, and C. Neighbors, "A tutorial on count regression and zero-altered count models for longitudinal substance use data.," Psychology of Addictive Behaviors, vol. 27 , no. 1, p. 166, 2013.

[65] J. L. Maggs, L. R. Williams, and C. M. Lee, "Ups and downs of alcohol use among first-year college students: Number of drinks, heavy drinking, and stumble and pass out drinking days," Addictive behaviors, vol. 36, no. 3, pp. 197-202, 2011.

[66] E. Tang, H. Ju, G. L. Baum, D. R. Roalf, T. D. Satterthwaite, F. Pasqualetti, and D. S. Bassett, "Control of brain network dynamics across diverse scales of space and time," Physical Review E, vol. 101, no. 6, p. 062301, 2020.

[67] D. K. Sherman, G. L. Cohen, L. D. Nelson, A. D. Nussbaum, D. P. Bunyan, and J. Garcia, "Affirmed yet unaware: exploring the role of awareness in the process of self-affirmation.," Journal of personality and social psychology, vol. 97, no. 5, p. 745, 2009.

[68] A. D’Argembeau, D. Stawarczyk, S. Majerus, F. Collette, M. Van der Linden, D. Feyers, P. Maquet, and E. Salmon, "The neural basis of personal goal processing when envisioning future events," Journal of cognitive neuroscience, vol. 22, no. 8, pp. 1701-1713, 2010.

[69] Y. Kang, N. Cooper, P. Pandey, C. Scholz, M. B. O’Donnell, M. D. Lieberman, S. E. Taylor, V. J. Strecher, S. Dal Cin, S. Konrath, et al., "Effects of self-transcendence on neural responses to persuasive messages and health behavior change," Proceedings of the National Academy of Sciences, vol. 115, no. 40, pp. 9974-9979, 2018. 
[70] L. N. Ross, "Causal concepts in biology: How pathways differ from mechanisms and why it matters," The British Journal for the Philosophy of Science, 2020.

[71] J. W. Schooler, J. Smallwood, K. Christoff, T. C. Handy, E. D. Reichle, and M. A. Sayette, "Metaawareness, perceptual decoupling and the wandering mind," Trends in cognitive sciences, vol. 15, no. 7, pp. 319-326, 2011.

[72] J. Bargh, "The four horsemen of automaticity: Awareness, intention, efficiency, and control in social cognition.," 1994.

[73] L. S. Hallion, A. M. Ruscio, and A. P. Jha, "Fractionating the role of executive control in control over worry: A preliminary investigation," Behaviour Research and Therapy, vol. 54, pp. 1-6, 2014.

[74] J. Nakamura and M. Csikszentmihalyi, "The concept of flow," in Flow and the foundations of positive psychology, pp. 239-263, Springer, 2014.

[75] Y. Kang, "Examining interpersonal self-transcendence as a potential mechanism linking meditation and social outcomes," Current opinion in psychology, vol. 28, pp. 115-119, 2019.

[76] J. A. Coan, C. L. Brown, and L. Beckes, "Our social baseline: The role of social proximity in economy of action.," 2014.

[77] L. Q. Uddin, "Cognitive and behavioural flexibility: neural mechanisms and clinical considerations," Nature Reviews Neuroscience, vol. 22, no. 3, pp. 167-179, 2021.

[78] D. S. Bassett, N. F. Wymbs, M. A. Porter, P. J. Mucha, J. M. Carlson, and S. T. Grafton, "Dynamic reconfiguration of human brain networks during learning," Proceedings of the National Academy of Sciences, vol. 108, no. 18, pp. 7641-7646, 2011.

[79] J. Chen, Y. C. Leong, C. J. Honey, C. H. Yong, K. A. Norman, and U. Hasson, "Shared memories reveal shared structure in neural activity across individuals," Nature neuroscience, vol. 20, no. 1, pp. 115-125, 2017.

[80] S. S. Cohen and C. Baldassano, "Developmental changes in story-evoked responses in the neocortex and hippocampus," bioRxiv, 2021.

[81] H. F. Chua, I. Liberzon, R. C. Welsh, and V. J. Strecher, "Neural correlates of message tailoring and self-relatedness in smoking cessation programming," Biological psychiatry, vol. 65, no. 2, pp. 165-168, 2009. 
[82] C. L. Boness, A. L. Watts, K. N. Moeller, and K. J. Sher, "The etiologic, theory-based, ontogenetic hierarchical framework of alcohol use disorder: A translational systematic review of reviews," 2021.

[83] E. J. Cornblath, A. Ashourvan, J. Z. Kim, R. F. Betzel, R. Ciric, A. Adebimpe, G. L. Baum, X. He, K. Ruparel, T. M. Moore, et al., "Temporal sequences of brain activity at rest are constrained by white matter structure and modulated by cognitive demands," Communications biology, vol. 3, no. 1, pp. 1-12, 2020.

[84] C. J. Honey, O. Sporns, L. Cammoun, X. Gigandet, J.-P. Thiran, R. Meuli, and P. Hagmann, "Predicting human resting-state functional connectivity from structural connectivity," Proceedings of the National Academy of Sciences, vol. 106, no. 6, pp. 2035-2040, 2009.

[85] E. Nozari, J. Stiso, L. Caciagli, E. J. Cornblath, X. He, M. A. Bertolero, A. S. Mahadevan, G. J. Pappas, and D. S. Bassett, "Is the brain macroscopically linear? a system identification of resting state dynamics," arXiv preprint arXiv:2012.12351, 2020.

[86] J. Peirce, J. R. Gray, S. Simpson, M. MacAskill, R. Höchenberger, H. Sogo, E. Kastman, and J. K. Lindeløv, "Psychopy2: Experiments in behavior made easy," Behavior research methods, vol. 51, no. 1, pp. 195-203, 2019.

[87] K. J. Gorgolewski, T. Auer, V. D. Calhoun, R. C. Craddock, S. Das, E. P. Duff, G. Flandin, S. S. Ghosh, T. Glatard, Y. O. Halchenko, et al., "The brain imaging data structure, a format for organizing and describing outputs of neuroimaging experiments," Scientific data, vol. 3, no. 1, pp. 1-9, 2016.

[88] Y. Halchenko, M. Goncalves, M. V. di Oleggio Castello, S. Ghosh, M. Hanke, M. Brett, T. Salo, C. F. Gorgolewski, J. Stadler, john lee, pvelasco, D. Lurie, J. Pellman, B. Poldrack, B. Schiffler, M. Szczepanik, and J. Carlin, "nipy/heudiconv: Heudiconv v0.5.1," July 2018.

[89] M. Cieslak, P. A. Cook, X. He, F.-C. Yeh, T. Dhollander, A. Adebimpe, G. K. Aguirre, D. S. Bassett, R. F. Betzel, J. Bourque, et al., "Qsiprep: an integrative platform for preprocessing and reconstructing diffusion mri data," Nature Methods, pp. 1-4, 2021.

[90] A. Schaefer, R. Kong, E. M. Gordon, T. O. Laumann, X.-N. Zuo, A. J. Holmes, S. B. Eickhoff, and B. T. Yeo, "Local-global parcellation of the human cerebral cortex from intrinsic functional connectivity mri," Cerebral cortex, vol. 28, no. 9, pp. 3095-3114, 2018. 
[91] S. M. Smith, M. Jenkinson, M. W. Woolrich, C. F. Beckmann, T. E. Behrens, H. Johansen-Berg, P. R. Bannister, M. De Luca, I. Drobnjak, D. E. Flitney, et al., "Advances in functional and structural mr image analysis and implementation as fsl," Neuroimage, vol. 23, pp. S208-S219, 2004.

[92] F.-C. Yeh, V. J. Wedeen, and W.-Y. I. Tseng, "Generalized q-sampling imaging," IEEE transactions on medical imaging, vol. 29, no. 9, pp. 1626-1635, 2010.

[93] F.-C. Yeh, T. D. Verstynen, Y. Wang, J. C. Fernández-Miranda, and W.-Y. I. Tseng, "Deterministic diffusion fiber tracking improved by quantitative anisotropy," PloS one, vol. 8, no. 11, p. e80713, 2013.

[94] K. Gorgolewski, C. D. Burns, C. Madison, D. Clark, Y. O. Halchenko, M. L. Waskom, and S. S. Ghosh, "Nipype: a flexible, lightweight and extensible neuroimaging data processing framework in python," Frontiers in neuroinformatics, vol. 5, p. 13, 2011.

[95] O. Esteban, C. J. Markiewicz, R. W. Blair, C. A. Moodie, A. I. Isik, A. Erramuzpe, J. D. Kent, M. Goncalves, E. DuPre, M. Snyder, et al., "fmriprep: a robust preprocessing pipeline for functional mri," Nature methods, vol. 16, no. 1, pp. 111-116, 2019.

[96] N. J. Tustison, B. B. Avants, P. A. Cook, Y. Zheng, A. Egan, P. A. Yushkevich, and J. C. Gee, "N4itk: improved n3 bias correction," IEEE transactions on medical imaging, vol. 29, no. 6, pp. 1310-1320, 2010.

[97] B. B. Avants, C. L. Epstein, M. Grossman, and J. C. Gee, "Symmetric diffeomorphic image registration with cross-correlation: evaluating automated labeling of elderly and neurodegenerative brain," Medical image analysis, vol. 12, no. 1, pp. 26-41, 2008.

[98] Y. Zhang, M. Brady, and S. Smith, "Segmentation of brain mr images through a hidden markov random field model and the expectation-maximization algorithm," IEEE Transactions on Medical Imaging, vol. 20, no. 1, pp. 45-57, 2001.

[99] A. M. Dale, B. Fischl, and M. I. Sereno, "Cortical surface-based analysis: I. segmentation and surface reconstruction," Neuroimage, vol. 9, no. 2, pp. 179-194, 1999.

[100] A. Klein, S. S. Ghosh, F. S. Bao, J. Giard, Y. Häme, E. Stavsky, N. Lee, B. Rossa, M. Reuter, E. Chaibub Neto, et al., "Mindboggling morphometry of human brains," PLoS computational biology, vol. 13, no. 2, p. e1005350, 2017. 
[101] V. S. Fonov, A. C. Evans, R. C. McKinstry, C. Almli, and D. Collins, "Unbiased nonlinear average age-appropriate brain templates from birth to adulthood," NeuroImage, no. 47, p. S102, 2009.

[102] R. W. Cox, "Afni: software for analysis and visualization of functional magnetic resonance neuroimages," Computers and Biomedical research, vol. 29, no. 3, pp. 162-173, 1996.

[103] D. N. Greve and B. Fischl, "Accurate and robust brain image alignment using boundary-based registration," Neuroimage, vol. 48, no. 1, pp. 63-72, 2009.

[104] M. Jenkinson, P. Bannister, M. Brady, and S. Smith, "Improved optimization for the robust and accurate linear registration and motion correction of brain images," Neuroimage, vol. 17, no. 2, pp. 825$841,2002$.

[105] R. Ciric, A. F. Rosen, G. Erus, M. Cieslak, A. Adebimpe, P. A. Cook, D. S. Bassett, C. Davatzikos, D. H. Wolf, and T. D. Satterthwaite, "Mitigating head motion artifact in functional connectivity mri," Nature protocols, vol. 13, no. 12, pp. 2801-2826, 2018.

[106] D. Cosme, J. Flournoy, and K. DeStasio, "dsnlab/auto-motion: In house," May 2018.

[107] R. W. Cox, G. Chen, D. R. Glen, R. C. Reynolds, and P. A. Taylor, "Fmri clustering in afni: falsepositive rates redux," Brain connectivity, vol. 7, no. 3, pp. 152-171, 2017.

[108] J. Veraart, D. S. Novikov, D. Christiaens, B. Ades-Aron, J. Sijbers, and E. Fieremans, "Denoising of diffusion mri using random matrix theory," Neuroimage, vol. 142, pp. 394-406, 2016.

[109] E. Kellner, B. Dhital, V. G. Kiselev, and M. Reisert, "Gibbs-ringing artifact removal based on local subvoxel-shifts," Magnetic resonance in medicine, vol. 76, no. 5, pp. 1574-1581, 2016.

[110] J. L. Andersson and S. N. Sotiropoulos, "An integrated approach to correction for off-resonance effects and subject movement in diffusion mr imaging," Neuroimage, vol. 125, pp. 1063-1078, 2016.

[111] J. L. Andersson, S. Skare, and J. Ashburner, "How to correct susceptibility distortions in spin-echo echo-planar images: application to diffusion tensor imaging," Neuroimage, vol. 20, no. 2, pp. 870-888, 2003.

[112] J. D. Power, A. Mitra, T. O. Laumann, A. Z. Snyder, B. L. Schlaggar, and S. E. Petersen, "Methods to detect, characterize, and remove motion artifact in resting state fmri," Neuroimage, vol. 84, pp. 320$341,2014$. 
[113] A. Abraham, F. Pedregosa, M. Eickenberg, P. Gervais, A. Mueller, J. Kossaifi, A. Gramfort, B. Thirion, and G. Varoquaux, "Machine learning for neuroimaging with scikit-learn," Frontiers in neuroinformatics, vol. 8, p. 14, 2014.

[114] E. Garyfallidis, M. Brett, B. Amirbekian, A. Rokem, S. Van Der Walt, M. Descoteaux, and I. NimmoSmith, "Dipy, a library for the analysis of diffusion mri data," Frontiers in neuroinformatics, vol. 8, p. 8,2014 .

[115] R. S. Desikan, F. Ségonne, B. Fischl, B. T. Quinn, B. C. Dickerson, D. Blacker, R. L. Buckner, A. M. Dale, R. P. Maguire, B. T. Hyman, et al., "An automated labeling system for subdividing the human cerebral cortex on mri scans into gyral based regions of interest," Neuroimage, vol. 31, no. 3, pp. 968-980, 2006.

[116] P. Srivastava, E. Nozari, J. Z. Kim, H. Ju, D. Zhou, C. Becker, F. Pasqualetti, G. J. Pappas, and D. S. Bassett, "Models of communication and control for brain networks: distinctions, convergence, and future outlook," Network Neuroscience, vol. 4, no. 4, pp. 1122-1159, 2020.

[117] A. S. Mahadevan, E. J. Cornblath, D. M. Lydon-Staley, D. J. Zhou, L. M. Parkes, B. Larsen, A. Adebimpe, A. E. Kahn, R. C. Gur, R. E. Gur, et al., "Alprazolam modulates persistence energy during emotion processing in first-degree relatives of individuals with schizophrenia: a network control study," bioRxiv, 2021.

[118] R. Gao, R. L. van den Brink, T. Pfeffer, and B. Voytek, "Neuronal timescales are functionally dynamic and shaped by cortical microarchitecture," Elife, vol. 9, p. e61277, 2020.

[119] M. E. Brooks, K. Kristensen, K. J. Van Benthem, A. Magnusson, C. W. Berg, A. Nielsen, H. J. Skaug, M. Machler, and B. M. Bolker, "glmmtmb balances speed and flexibility among packages for zero-inflated generalized linear mixed modeling," The R journal, vol. 9, no. 2, pp. 378-400, 2017.

[120] J. W. LaBrie, J. F. Hummer, and E. R. Pedersen, "Reasons for drinking in the college student context: The differential role and risk of the social motivator," Journal of studies on alcohol and drugs, vol. 68, no. 3, pp. 393-398, 2007.

[121] K. L. Gratz and L. Roemer, "Multidimensional assessment of emotion regulation and dysregulation: Development, factor structure, and initial validation of the difficulties in emotion regulation scale," Journal of psychopathology and behavioral assessment, vol. 26, no. 1, pp. 41-54, 2004. 
[122] S. M. Mitchell, S. Lange, and H. Brus, "Gendered citation patterns in international relations journals," International Studies Perspectives, vol. 14, no. 4, pp. 485-492, 2013.

[123] M. L. Dion, J. L. Sumner, and S. M. Mitchell, "Gendered citation patterns across political science and social science methodology fields," Political Analysis, vol. 26, no. 3, pp. 312-327, 2018.

[124] N. Caplar, S. Tacchella, and S. Birrer, "Quantitative evaluation of gender bias in astronomical publications from citation counts," Nature Astronomy, vol. 1, no. 6, p. 0141, 2017.

[125] D. Maliniak, R. Powers, and B. F. Walter, "The gender citation gap in international relations," International Organization, vol. 67, no. 4, pp. 889-922, 2013.

[126] J. D. Dworkin, K. A. Linn, E. G. Teich, P. Zurn, R. T. Shinohara, and D. S. Bassett, "The extent and drivers of gender imbalance in neuroscience reference lists," bioRxiv, 2020.

[127] M. A. Bertolero, J. D. Dworkin, S. U. David, C. L. Lloreda, P. Srivastava, J. Stiso, D. Zhou, K. Dzirasa, D. A. Fair, A. N. Kaczkurkin, B. J. Marlin, D. Shohamy, L. Q. Uddin, P. Zurn, and D. S. Bassett, "Racial and ethnic imbalance in neuroscience reference lists and intersections with gender," bioRxiv, 2020.

[128] X. Wang, J. D. Dworkin, D. Zhou, J. Stiso, E. B. Falk, D. S. Bassett, P. Zurn, and D. M. Lydon-Staley, "Gendered citation practices in the field of communication," Annals of the International Communication Association, 2021.

[129] P. Chatterjee and R. M. Werner, "Gender disparity in citations in high-impact journal articles," JAMA Netw Open, vol. 4, no. 7, p. e2114509, 2021.

[130] J. M. Fulvio, I. Akinnola, and B. R. Postle, "Gender (im)balance in citation practices in cognitive neuroscience," J Cogn Neurosci, vol. 33, no. 1, pp. 3-7, 2021.

[131] D. Zhou, E. J. Cornblath, J. Stiso, E. G. Teich, J. D. Dworkin, A. S. Blevins, and D. S. Bassett, "Gender diversity statement and code notebook v1.0," Feb. 2020.

[132] A. Ambekar, C. Ward, J. Mohammed, S. Male, and S. Skiena, "Name-ethnicity classification from open sources," in Proceedings of the 15th ACM SIGKDD international conference on Knowledge Discovery and Data Mining, pp. 49-58, 2009. 
[133] G. Sood and S. Laohaprapanon, "Predicting race and ethnicity from the sequence of characters in a name," arXiv preprint arXiv:1805.02109, 2018. 